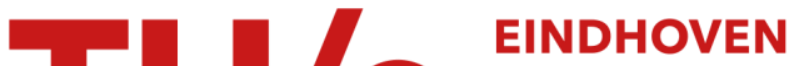 UNIVERSITY OF TECHNOLOGY
}

\section{Frequency Logarithmic Perturbation on the Group-Velocity Dispersion Parameter With Applications to Passive Optical Networks}

Citation for published version (APA):

Oliari, V., Agrell, E., Liga, G., \& Alvarado, A. (2021). Frequency Logarithmic Perturbation on the Group-Velocity Dispersion Parameter With Applications to Passive Optical Networks. Journal of Lightwave Technology, 39(16), 5287-5299. [9502568]. https://doi.org/10.1109/JLT.2021.3101055

\section{Document license:}

TAVERNE

DOI:

10.1109/JLT.2021.3101055

Document status and date:

Published: 30/07/2021

\section{Document Version:}

Publisher's PDF, also known as Version of Record (includes final page, issue and volume numbers)

\section{Please check the document version of this publication:}

- A submitted manuscript is the version of the article upon submission and before peer-review. There can be important differences between the submitted version and the official published version of record. People interested in the research are advised to contact the author for the final version of the publication, or visit the DOI to the publisher's website.

- The final author version and the galley proof are versions of the publication after peer review.

- The final published version features the final layout of the paper including the volume, issue and page numbers.

Link to publication

\footnotetext{
General rights

- You may freely distribute the URL identifying the publication in the public portal. follow below link for the End User Agreement:

www.tue.nl/taverne

\section{Take down policy}

If you believe that this document breaches copyright please contact us at:

openaccess@tue.nl

providing details and we will investigate your claim.
}

Copyright and moral rights for the publications made accessible in the public portal are retained by the authors and/or other copyright owners and it is a condition of accessing publications that users recognise and abide by the legal requirements associated with these rights.

- Users may download and print one copy of any publication from the public portal for the purpose of private study or research.

- You may not further distribute the material or use it for any profit-making activity or commercial gain

If the publication is distributed under the terms of Article $25 \mathrm{fa}$ of the Dutch Copyright Act, indicated by the "Taverne" license above, please 


\title{
Frequency Logarithmic Perturbation on the Group-Velocity Dispersion Parameter With Applications to Passive Optical Networks
}

\author{
Vinícius Oliari ${ }^{\circledR}$, Graduate Student Member, IEEE, Erik Agrell ${ }^{\circledR}$, Fellow, IEEE, Gabriele Liga ${ }^{\circledR}$, Member, IEEE, \\ and Alex Alvarado ${ }^{(D)}$, Senior Member, IEEE
}

(Top-Scored Paper)

\begin{abstract}
Signal propagation in an optical fiber can be described by the nonlinear Schrödinger equation (NLSE). The NLSE has no known closed-form solution when both dispersion and nonlinearities are considered simultaneously. In this paper, we present a novel integral-form approximate model for the nonlinear optical channel, with applications to passive optical networks. The proposed model is derived using logarithmic perturbation in the frequency domain on the group-velocity dispersion (GVD) parameter of the NLSE. The model can be seen as an improvement of the recently proposed regular perturbation (RP) on the GVD parameter. RP and logarithmic perturbation (LP) on the nonlinear coefficient have already been studied in the literature, and are hereby compared with RP on the GVD parameter and the proposed LP model. As an application of the model, we focus on passive optical networks. For a $20 \mathrm{~km}$ PON at 10 Gbaud, the proposed model improves the normalized square deviation by $1.5 \mathrm{~dB}$ with respect to $\mathrm{LP}$ on the nonlinear coefficient. For the same system, histogram-based detectors are developed using the received symbols from the models. The detector obtained from the proposed LP model reduces the uncoded bit-error-rate by up to 5.4 times at the same input power or reduces the input power by $0.4 \mathrm{~dB}$ at the same information rate compared to the detector obtained from LP on the nonlinear coefficient.
\end{abstract}

Index Terms-Channel modeling, chromatic dispersion, Kerr nonlinearity, logarithmic perturbation, nonlinear Schrödinger equation, optical fiber, regular perturbation, weakly dispersive regime.

Manuscript received March 9, 2021; revised June 28, 2021; accepted July 21, 2021. Date of publication July 30, 2021; date of current version August 30, 2021. This work is supported by the Netherlands Organisation for Scientific Research (NWO) via the VIDI Grant ICONIC under Grant 15685. The work of A. Alvarado has received funding from the European Research Council (ERC) under the European Union's Horizon 2020 research and innovation programme under Grant 757791. The work of E. Agrell has received funding from the Swedish Research Council (VR) under Grant 2017-03702. The work of G. Liga is supported by the EuroTechPostdoc programme under the European Union's Horizon 2020 research and innovation programme (Marie Skłodowska-Curie) Grant 754462. (Corresponding author: Vinícius Oliari.)

Vinícius Oliari, Gabriele Liga, and Alex Alvarado are with Signal Processing Systems (SPS) Group, Department of Electrical Engineering, Eindhoven University of Technology, Eindhoven, MB 5600, The Netherlands (e-mail: v.oliari.couto.dias@tue.nl; g.liga@tue.nl; a.alvarado@tue.nl).

Erik Agrell is with the Department of Electrical Engineering, Chalmers University of Technology, Gothenburg SE-41296, Sweden (e-mail: agrell@ chalmers.se).

Color versions of one or more figures in this article are available at https: //doi.org/10.1109/JLT.2021.3101055.

Digital Object Identifier 10.1109/JLT.2021.3101055

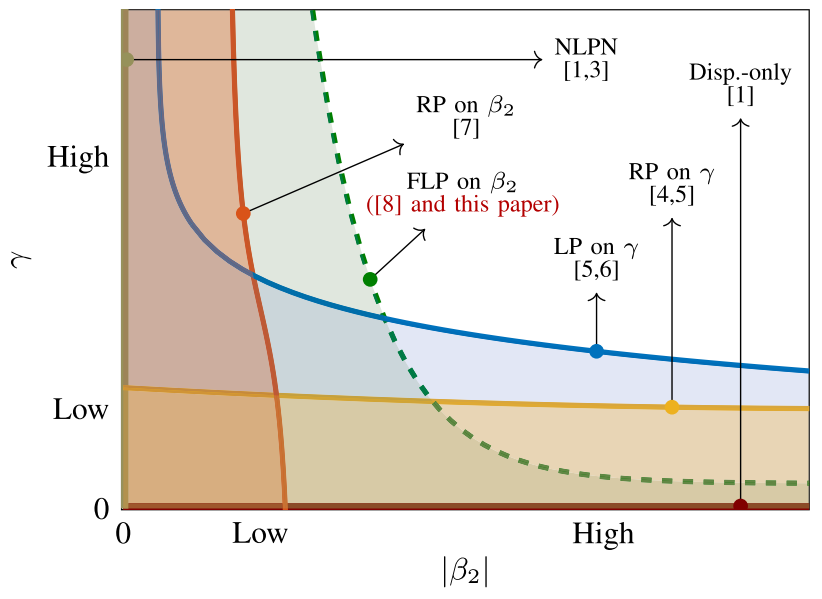

Fig. 1. Illustration of the regions in the $\beta_{2}$ vs $\gamma$ plane where different models are valid. This paper presents a new model for the region to the left of and under the green curve

\section{INTRODUCTION}

A NALYTICAL models for optical fiber transmission have been widely studied in the literature. These models are based on the equations that govern the optical field propagation: the nonlinear Schrödinger equation (NLSE) [1, Ch. 2] and its variants. The NLSE has no known exact solution for an arbitrary input waveform. One of the most efficient alternatives for approximated numerical solutions is the split step Fourier method (SSFM) [2], which simulates the effects of fiber propagation. On the other hand, to analyse these effects and design novel transceivers, analytical models are highly desirable.

Some of the NLSE's most used analytical models are only valid under some restricted values of two fiber parameters: the (Kerr) nonlinear coefficient $\gamma$ and the group-velocity dispersion (GVD) parameter $\beta_{2}$. The validity ${ }^{1}$ of the models with respect to these parameters is shown in Fig. 1. When $\gamma$ is equal to zero, the NLSE admits an analytical solution, given by the dispersiononly model [1, Ch. 3] and represented by the horizontal line at

\footnotetext{
${ }^{1}$ The validity of a model is defined in this paper as the set of parameters values in which the model waveform is sufficiently close to the true waveform obtained from the NLSE. Details on the metric that measures the distance between the two waveforms are given in Section III.
} 
$\gamma=0$ in Fig. 1. When $\beta_{2}$ is set to zero, the NLSE also admits an analytical solution, given by the nonlinear phase noise model (NLPN) [1, Ch. 4], [3], represented by the vertical line at $\beta_{2}=0$ in Fig. 1. If both parameters are nonzero, usually a perturbation approach is used [4].

A perturbation on the nonlinear coefficient $\gamma$ considers the nonlinearities a minor effect [4], [5] and is accurate for high accumulated dispersion, where the accumulated dispersion can increase with the signal bandwidth and with the product of $\beta_{2}$ with the fiber length. The development of a perturbation on $\gamma$, specifically the regular perturbation (RP) on $\gamma$, was an important mark in the literature since it has been used in many applications. For example, RP on $\gamma$ is a key step in the derivation of the Gaussian noise [6] and enhanced Gaussian noise [7] models. These two latter models have been widely used to estimate system performance, even in the presence of effects such as stimulated Raman scattering [8]. RP on $\gamma$ has also been used in the literature to build low-complexity receivers [9].

For the opposite regime, where the nonlinearities are the major effect and the accumulated dispersion is low but nonzero, a perturbation on the GVD parameter can be performed. The perturbation techniques usually considered for the optical fiber propagation are RP and logarithmic perturbation (LP) [4]. It was shown in [10] that LP converges faster to the true NLSE's solution than RP. RP and LP on $\gamma$ cover the area under the yellow and blue curves in Fig. 1, respectively. Recently, we proposed RP on $\beta_{2}$ in [11] and compared with RP on $\gamma$. RP on $\beta_{2}$ provided more accuracy in the weakly dispersive and highly nonlinear regimes, represented by the area under the red curve in Fig. 1. A preliminary investigation of LP on $\beta_{2}$ was reported in our recent work [12], where LP methods on both $\gamma$ and $\beta_{2}$ were compared on the waveform level. This paper is an extension of [12], to which we add two more contributions. Our first contribution is to derive the two perturbative models on $\beta_{2}$ presented in [12]. These models are obtained applying LP in either time or frequency. The latter approach, which we call frequency logarithmic perturbation (FLP), is the most accurate of the two for the $\beta_{2}$ expansion.

The FLP on $\beta_{2}$ covers the area under the green dashed curve in Fig. 1, which improves upon RP on $\beta_{2}$. FLP should not be confused with the frequency resolved logarithmic perturbation (FRLP) proposed in [13]-[15]. FRLP consists in applying LP on the frequency components of the time-domain signal individually and using the superposition principle to combine them. However, FRLP still applies the LP principle in the time domain, whereas FLP we study here applies this principle in the frequency domain. In this paper, the FLP on $\beta_{2}$ model is compared with the LP on $\gamma$, RP on $\gamma$, and RP on $\beta_{2}$ models.

The second contribution of this paper is to demonstrate the applicability of the proposed FLP on $\beta_{2}$ model. To this end, we consider passive optical networks (PONs), wherein the accumulated dispersion is typically low. PONs are usually short-reach fiber links where the transmitted signal is split to numerous users [16]. The input power can increase significantly when the split ratio is high, in order to compensate for the split loss [17], [18]. At a high input powers, the transmission can enter a highly-nonlinear regime, where even pre-distortion using digital backpropagation based on the SSFM has been proposed in the literature [19].

We use the models in a PON system to estimate optimum decision regions at the receiver. Simulations are carried out in the $\mathrm{C}$ and O-band, where the latter has a close-to-zero GVD parameter $\beta_{2}$. At a waveform level (continuous-time), the proposed FLP on $\beta_{2}$ is shown to outperform the other three models at powers higher than $7 \mathrm{dBm}$ for both $\mathrm{C}$ - and O-band systems. At a symbol level (discrete-time), decision regions obtained from LP on $\beta_{2}$ result in bit-error-rates (BER) more than five times lower than the ones obtained from LP on $\gamma$. Finally, these decision regions are analysed in a system with forward-error-correction (FEC).

This paper is organized as follows: Section II provides the mathematical background for the derivation of the models; Section III compares the models in both continuous- and discretetime; and Section IV concludes the paper.

\section{MATHEMATICAL BACKGROUND}

The NLSE normalized by the attenuation factor for noiseless propagation of an optical field $A$ at a retarded time frame $t$ and distance $z$ for a single-polarization can be approximated as [5]

$$
\begin{aligned}
\frac{\partial A(t, z)}{\partial z}= & -\frac{j \beta_{2}}{2} \frac{\partial^{2} A(t, z)}{\partial t^{2}} \\
& +j \gamma \mathrm{e}^{-\alpha z}|A(t, z)|^{2} A(t, z),
\end{aligned}
$$

where $\alpha$ is the attenuation coefficient, $\beta_{2}$ the GVD parameter, and $\gamma$ the nonlinear coefficient. The first term on the right-hand side of (1) represents the chromatic dispersion. This effect on the waveform $A$ is larger when the bandwidth and/or the fiber length is increased. The last term represents the Kerr nonlinearity, which has a cubic dependence on the instantaneous signal power and also increases with the fiber length. The solution $A$ of (1) can be numerically estimated by the SSFM [1, Ch. 2], [2]. Other effects, such as third-order dispersion (TOD) [1, Ch. 3], are not taken into account in (1). TOD becomes significant for large bandwidths or when $\beta_{2}$ is low. This effect will be taken into account for our simulations in the O-band, although it is not used in the derivation of the proposed model. For the C-band, we consider solely the effects in (1).

In what follows, we first review three models available in the literature. Section II-A and Section II-B describe the RP on $\gamma$ and on $\beta_{2}$, respectively, while Section II-C presents the LP on $\gamma$. Finally, Section II-D introduces the FLP on $\beta_{2}$.

\section{A. Regular Perturbation on the Nonlinear Coefficient}

The RP on $\gamma$ was first derived in [4], [5]. To approximate the solution of (1), the RP method represents the signal by a power series of a certain coefficient. For the RP on $\gamma$, the RP solution can be written as

$$
A(t, z)=\sum_{k=0}^{\infty} \gamma^{k} A_{k}^{(\gamma)}(t, z)
$$

To obtain the functions $A_{k}^{(\gamma)}$, (2) is substituted into (1) and the terms multiplied by the $k$-th power of $\gamma$ are equated. An approximate solution for (1) can be obtained by considering only the functions $A_{0}^{(\gamma)}$ and $A_{1}^{(\gamma)}$. This approximation is the 
first-order RP on $\gamma$ [5, Eqs. (7), (9)], [4, Eq. (12)]

$$
A(t, z) \approx A_{\mathrm{RP}}^{(\gamma)}(t, z)=A_{0}^{(\gamma)}(t, z)+\gamma A_{1}^{(\gamma)}(t, z),
$$

where

$$
\begin{aligned}
& A_{0}^{(\gamma)}(t, z)=\mathcal{D}_{z}\{A(\cdot, 0)\}(t), \\
& A_{1}^{(\gamma)}(t, z)=j \int_{0}^{z} \mathrm{e}^{-\alpha u} \mathcal{D}_{z-u}\left\{\left|A_{0}(\cdot, u)\right|^{2} A_{0}(\cdot, u)\right\}(t) \mathrm{d} u,
\end{aligned}
$$

and the dispersion operator $\mathcal{D}_{z}$ is

$$
\begin{aligned}
\mathcal{D}_{z}\{f\}(t) & \triangleq(f * h(\cdot, z))(t), \\
h(t, z) & =\frac{1}{\sqrt{j 2 \pi \beta_{2} z}} \mathrm{e}^{-\frac{j}{2 \beta_{2} z}} t^{2} .
\end{aligned}
$$

The function $A_{0}^{(\gamma)}$ in (4) is called the dispersion-only solution of (1). This solution can be seen as a model that is accurate only when the nonlinear effect is negligible. The first-order RP on $\gamma$ in (3) is accurate for low nonlinear effects and is illustrated by the yellow curve in Fig. 1.

The RP on $\gamma$ also benefits from a simple mathematical trick that yields an increased accuracy. This trick was developed in [5] and is based on modifying (1) by making

$$
A(t, z)=A_{P}(t, z) e^{j \gamma P_{0} G(z)},
$$

where $G(z)=(1-\exp (-\alpha z)) / \alpha$. Although $P_{0}$ was defined in [5] as the peak input power, we use $P_{0}$ as the average input power, which yields better accuracy in our simulations. After substituting (8) in (1), one can apply the RP on $\gamma$ method over the function $A_{P}$ instead of $A$ and obtain the first order RP approximation for $A_{P}$. Multiplying the obtained approximation by $e^{j \gamma P_{0} G(z)}$, as in (8), yields

$$
\begin{aligned}
A(t, z) & \approx A_{\mathrm{ERP}}^{(\gamma)}(t, z) \\
& =\left[\left(1-j \gamma P_{0} G(z)\right) A_{0}^{(\gamma)}(t, z)+\gamma A_{1}^{(\gamma)}(t, z)\right] e^{j \gamma P_{0} G(z)},
\end{aligned}
$$

where $A_{0}^{(\gamma)}$ and $A_{1}^{(\gamma)}$ are given by (4) and (5), respectively. Another benefit from RP on $\gamma$ is that it can easily generate a solution that also accounts for TOD. This new solution is derived from (1) with an additional term accounting for TOD [1, Eq. (3.3.1)]

$$
\begin{aligned}
\frac{\partial A(t, z)}{\partial z}= & -\frac{j \beta_{2}}{2} \frac{\partial^{2} A(t, z)}{\partial t^{2}}+\frac{\beta_{3}}{6} \frac{\partial^{3} A(t, z)}{\partial t^{3}} \\
& +j \gamma \mathrm{e}^{-\alpha z}|A(t, z)|^{2} A(t, z) .
\end{aligned}
$$

The RP on $\gamma$ with TOD is then obtained by replacing the operator $\mathcal{D}_{z}$ in (4) and (5) by

$$
\begin{aligned}
\mathcal{T}_{z}\{f\}(t) & \triangleq \mathcal{F}^{-1}\{\tilde{T}(\cdot, z) \cdot \tilde{f}\}(t), \\
\tilde{T}(\omega, z) & =e^{\left(\frac{j \beta_{2}}{2} \omega^{2}+\frac{j \beta_{3}}{6} \omega^{3}\right) z},
\end{aligned}
$$

where $\mathcal{F}^{-1}$ denotes the inverse Fourier transform ${ }^{2}, \omega$ is the angular frequency, and $\tilde{f}$ is the Fourier transform of the function $f$.

${ }^{2}$ We define the Fourier transform of a function $A(\cdot, z)$ as $\tilde{A}(\omega, z) \triangleq$ $\int_{-\infty}^{\infty} A(t, z) \mathrm{e}^{+j \omega t} \mathrm{~d} t$, which depends on the angular frequency $\omega$ and is evaluated at distance $z$. The inverse Fourier transform of $\tilde{A}(\cdot, z)$ is $A(t, z)=$ $[1 /(2 \pi)] \int_{-\infty}^{\infty} \tilde{A}(\omega, z) \mathrm{e}^{-j \omega t} \mathrm{~d} \omega$.

\section{B. Regular Perturbation on the GVD Parameter}

We recently proposed the RP on $\beta_{2}$ in [11]. The same procedure as in (2) can be applied by considering $A$ as a power series of $\beta_{2}$, i.e.,

$$
A(t, z)=\sum_{k=0}^{\infty} \beta_{2}^{k} A_{k}^{\left(\beta_{2}\right)}(t, z) .
$$

In analogy to RP on $\gamma$, the functions $A_{k}^{\left(\beta_{2}\right)}$ are also obtained by replacing (13) in (1) and equating the terms related to the $k$-th power of $\beta_{2}$. For the first-order RP, involving the functions $A_{0}^{\left(\beta_{2}\right)}$ and $A_{1}^{\left(\beta_{2}\right)}$, an approximate solution for $A$ can be obtained by

$$
A(t, z) \approx A_{\mathrm{RP}}^{\left(\beta_{2}\right)}(t, z)=A_{0}^{\left(\beta_{2}\right)}(t, z)+\beta_{2} A_{1}^{\left(\beta_{2}\right)}(t, z),
$$

where

$$
A_{0}^{\left(\beta_{2}\right)}(t, z)=A(t, 0) \mathrm{e}^{j \gamma|A(t, 0)|^{2} G(z)},
$$

and

$$
A_{1}^{\left(\beta_{2}\right)}(t, z)=B(t, z) \mathrm{e}^{j \gamma|A(t, 0)|^{2} G(z)},
$$

with $B$ given by

$$
\begin{aligned}
B(t, z)= & -M(t) z+G_{1}(z) R(t)+G_{2}(z) P(t) \\
& -2 j \gamma A(t, 0) \Re\left\{A^{*}(t, 0) V(t, z)\right\} \\
V(t, z)= & G(z)\left[M(t) z-G_{1}(z) R(t)-G_{2}(z) P(t)\right] \\
& -G_{1}(z) M(t)+G_{2}(z) R(t)+G_{3}(z) P(t) \\
M(t)= & \frac{j}{2} \frac{\partial^{2} A(t, 0)}{\partial t^{2}} \\
R(t)= & \frac{\gamma}{2} A(t, 0) \frac{\partial^{2}|A(t, 0)|^{2}}{\partial t^{2}}+\gamma \frac{\partial A(t, 0)}{\partial t} \frac{\partial|A(t, 0)|^{2}}{\partial t} \\
P(t)= & \frac{j \gamma^{2}}{2} A(t, 0)\left(\frac{\partial|A(t, 0)|^{2}}{\partial t}\right)^{2} \\
G_{1}(z)= & \frac{\alpha z+\mathrm{e}^{-\alpha z}-1}{\alpha^{2}}, \\
G_{2}(z)= & \frac{2 \alpha z+4 \mathrm{e}^{-\alpha z}-\mathrm{e}^{-2 \alpha z}-3}{2 \alpha^{3}} \\
G_{3}(z)= & \frac{6 \alpha z+18 \mathrm{e}^{-\alpha z}-9 \mathrm{e}^{-2 \alpha z}+2 \mathrm{e}^{-3 \alpha z}-11}{6 \alpha^{4}} .
\end{aligned}
$$

Analogously to RP on $\gamma$, the function $A_{0}^{\left(\beta_{2}\right)}$ in (15) for RP on $\beta_{2}$ is an accurate model when dispersion is negligible, and is called the NLPN model [3], [11]. The first-order RP on $\beta_{2}$ in (14) is accurate for low accumulated dispersion and is illustrated as the red curve in Fig. 1.

\section{Logarithmic Perturbation}

LP is a mathematical technique similar to RP. LP on $\gamma$ was first presented in [4], [10] and can be shown to converge faster to the true NLSE's solution than RP on $\gamma$. LP functions can be obtained directly by the RP functions $A_{k}$. For example, following an approach similar to [4], consider that the signal $A$ can be written 
as a power series of a coefficient $\theta$ (e.g., $\gamma$ or $\beta_{2}$ as done in (2) and (13)) as

$$
A(t, z)=\sum_{k=0}^{\infty} \theta^{k} A_{k}^{(\theta)}(t, z)
$$

where $A_{k}^{(\theta)}$ is the $k$-th RP function. We now want to express $A$ in its LP version, which takes the form

$$
A(t, z)=A_{0}^{(\theta)}(t, z) \exp \left(\sum_{k=1}^{\infty} \theta^{k} \psi_{k}^{(\theta)}(t, z)\right),
$$

where the function $\psi_{k}^{(\theta)}$ is the $k$-th LP function and $A_{0}^{(\theta)}$ is the 0 -th order RP function. Representing the exponential function in (26) by its Taylor expansion yields

$$
A(t, z)=A_{0}^{(\theta)}(t, z) \sum_{m=0}^{\infty} \frac{1}{m !}\left(\sum_{k=1}^{\infty} \theta^{k} \psi_{k}^{(\theta)}(t, z)\right)^{m} .
$$

The functions $\psi_{k}^{(\theta)}$ can now be obtained by equating (27) with (25), and further equating the terms that have the same power of $\theta$. For example, equating the terms multiplied by $\theta^{1}$, we obtain the first-order LP function as

$$
\psi_{1}^{(\theta)}(t, z)=\frac{A_{1}^{(\theta)}(t, z)}{A_{0}^{(\theta)}(t, z)} .
$$

Equating the terms multiplied by $\theta^{2}$ we can also obtain the second-order LP function

$$
\psi_{2}^{(\theta)}(t, z)=\frac{A_{2}^{(\theta)}(t, z)}{A_{0}^{(\theta)}(t, z)}-\frac{1}{2}\left(\frac{A_{1}^{(\theta)}(t, z)}{A_{0}^{(\theta)}(t, z)}\right)^{2} .
$$

The function $\psi_{2}^{(\theta)}$ in (29) depends on the RP term $A_{2}^{(\theta)}$. For RP on $\gamma, A_{2}^{(\gamma)}$ is well defined [4, Eq. (12)], [5, Eq. (11)]. However, for RP on $\beta_{2}, A_{2}^{\left(\beta_{2}\right)}$ is not known in the literature at the time this paper is being written. Thus, we will restrict the analysis to first-order LP and RP only.

Setting $\theta=\gamma$ or $\theta=\beta_{2}$ in (26) and (28) and truncating the sum in (26) at $k=1$, we obtain the first-order LP on $\gamma$ and on $\beta_{2}$, respectively. The first-order LP on $\gamma$ is written as

$$
A(t, z) \approx A_{\mathrm{LP}}^{(\gamma)}(t, z)=A_{0}^{(\gamma)}(t, z) \exp \left(\gamma \frac{A_{1}^{(\gamma)}(t, z)}{A_{0}^{(\gamma)}(t, z)}\right),
$$

where $A_{0}^{(\gamma)}$ and $A_{1}^{(\gamma)}$ are given by (4) and (5), respectively. The accuracy of $A_{\mathrm{LP}}^{(\gamma)}$ is qualitatively illustrated by the blue curve in Fig. 1. The first-order LP on $\beta_{2}$ is similarly obtained as

$$
A(t, z) \approx A_{\mathrm{LP}}^{\left(\beta_{2}\right)}(t, z)=A_{0}^{\left(\beta_{2}\right)}(t, z) \exp \left(\beta_{2} \frac{A_{1}^{\left(\beta_{2}\right)}(t, z)}{A_{0}^{\left(\beta_{2}\right)}(t, z)}\right),
$$

where $A_{0}^{\left(\beta_{2}\right)}$ and $A_{1}^{\left(\beta_{2}\right)}$ are given by (15) and (16), respectively.

\section{Frequency Logarithmic Perturbation}

The linearity of (25) with respect to the functions $A_{k}^{(\theta)}$ suggests another approach to obtain a different LP solution. The new approach consists of performing the same steps as in (25)-(28) in the frequency domain, which we refer to as FLP. To obtain the FLP solution, we first express (25) in the frequency domain, i.e.,

$$
\tilde{A}(\omega, z)=\sum_{k=0}^{\infty} \theta^{k} \tilde{A}_{k}^{(\theta)}(\omega, z),
$$

where $\tilde{A}$ represents the Fourier transform of $A$ and $\omega$ is the angular frequency. Analogous to (26), now $\tilde{A}$ is expressed in its FLP version as

$$
\tilde{A}(\omega, z)=\tilde{A}_{0}^{(\theta)}(\omega, z) \exp \left(\sum_{k=1}^{\infty} \theta^{k} \tilde{\zeta}_{k}^{(\theta)}(\omega, z)\right),
$$

where the function $\tilde{\zeta}_{k}^{(\theta)}$ is the $k$-th FLP function and $\tilde{A}_{0}^{(\theta)}$ is the Fourier transform of the 0-th order RP function.

In complete analogy with the procedure used to obtain (28), the first-order FLP function is

$$
\tilde{\zeta}_{1}^{(\theta)}(\omega, z)=\frac{\tilde{A}_{1}^{(\theta)}(\omega, z)}{\tilde{A}_{0}^{(\theta)}(\omega, z)},
$$

which is used to obtain the first-order FLP on $\gamma$ and on $\beta_{2}$. The first-order FLP on $\gamma$ is

$$
\tilde{A}(\omega, z) \approx \tilde{A}_{\mathrm{FLP}}^{(\gamma)}(\omega, z)=\tilde{A}_{0}^{(\gamma)}(\omega, z) \exp \left(\gamma \frac{\tilde{A}_{1}^{(\gamma)}(\omega, z)}{\tilde{A}_{0}^{(\gamma)}(\omega, z)}\right),
$$

where $\tilde{A}_{0}^{(\gamma)}$ and $\tilde{A}_{1}^{(\gamma)}$ are the Fourier transforms of (4) and (5), respectively. The first-order FLP on $\beta_{2}$ is given by

$$
\tilde{A}(\omega, z) \approx \tilde{A}_{\mathrm{FLP}}^{\left(\beta_{2}\right)}(\omega, z)=\tilde{A}_{0}^{\left(\beta_{2}\right)}(\omega, z) \exp \left(\beta_{2} \frac{\tilde{A}_{1}^{\left(\beta_{2}\right)}(\omega, z)}{\tilde{A}_{0}^{\left(\beta_{2}\right)}(\omega, z)}\right),
$$

where $\tilde{A}_{0}^{\left(\beta_{2}\right)}$ and $\tilde{A}_{1}^{\left(\beta_{2}\right)}$ are the Fourier transforms of (15) and (16), respectively. The qualitative behaviour of the accuracy of $\tilde{A}_{\mathrm{FLP}}^{\left(\beta_{2}\right)}$ is illustrated as the green dashed curve in Fig. 1.

The functions $\tilde{\zeta}_{k}^{(\theta)}$ in (33) differ from the Fourier transform of $\psi_{k}^{(\theta)}$ in (26), since the exponential of the LP method was applied in the frequency domain. The expressions in (28) and (34) are the simplest example of this fact, since they do not form, in general, a Fourier transform pair. Therefore, we expect that these LP-based models result in a different accuracy for each perturbation coefficient $\left(\gamma\right.$ or $\left.\beta_{2}\right)$. As it will be seen later in Section III, $A_{\mathrm{LP}}^{(\gamma)}$ is more accurate than $A_{\mathrm{FLP}}^{(\gamma)}$, while $A_{\mathrm{FLP}}^{\left(\beta_{2}\right)}$ is more accurate than $A_{\mathrm{LP}}^{\left(\beta_{2}\right)}$. We believe that the difference between $\beta_{2}$ and $\gamma$ when comparing LP and FLP could be explained by the solution of (1) for only the chromatic dispersion effect or only the Kerr effect [1]. The solution for the chromatic dispersion effect only is an exponential in the frequency domain, which resembles the FLP approach. Similarly, the solution for the Kerr effect only is an exponential in the time domain, which resembles the LP approach.

Calculating the waveforms for both LP and FLP leads to a numerical issue related to the ratio in (28) and (34). When the denominator in one of those two equations tends to zero, the 
TABLE I

Summary of the First-Order Perturbation Methods Discussed in This PAPER

\begin{tabular}{|c|c|c|c|c|c|c|}
\hline \multirow[b]{2}{*}{ Coeff. $\theta$} & \multicolumn{6}{|c|}{ Perturbation method } \\
\hline & \multicolumn{2}{|c|}{$\begin{array}{c}1^{\mathrm{st}} \text { order RP } \\
A(t, z)=A_{0}^{(\theta)}(t, z)+\theta A_{1}^{(\theta)}(t, z)\end{array}$} & \multicolumn{2}{|c|}{$\begin{array}{c}1^{\text {st }} \text { order LP } \\
A(t, z)=A_{0}^{(\theta)}(t, z) \exp \left(\theta \psi_{1}^{(\theta)}(t, z)\right)\end{array}$} & \multicolumn{2}{|c|}{$\begin{array}{c}1^{\text {st }} \text { order FLP } \\
\tilde{A}(\omega, z)=\tilde{A}_{0}^{(\theta)}(\omega, z) \exp \left(\theta \tilde{\zeta}_{1}^{(\theta)}(\omega, z)\right)\end{array}$} \\
\hline \multirow{2}{*}{$\gamma$} & $A_{0}^{(\gamma)}(t, z)$ & $\mathcal{D}_{z}\{A(\cdot, 0)\}(t)$ & \multirow{2}{*}{$\psi_{1}^{(\gamma)}(t, z)$} & \multirow{2}{*}{$\frac{A_{1}^{(\gamma)}(t, z)}{A_{0}^{(\gamma)}(t, z)}$} & \multirow{2}{*}{$\tilde{\zeta}_{1}^{(\gamma)}(\omega, z)$} & \multirow{2}{*}{$\frac{\tilde{A}_{1}^{(\gamma)}(\omega, z)}{\tilde{A}_{0}^{(\gamma)}(\omega, z)}$} \\
\hline & $A_{1}^{(\gamma)}(t, z)$ & (5) & & & & \\
\hline \multirow{2}{*}{$\beta_{2}$} & $A_{0}^{\left(\beta_{2}\right)}(t, z)$ & $A(t, 0) \mathrm{e}^{j \gamma|A(t, 0)|^{2} G(z)}$ & \multirow{2}{*}{$\psi_{1}^{\left(\beta_{2}\right)}(t, z)$} & \multirow{2}{*}{$\frac{A_{1}^{\left(\beta_{2}\right)}(t, z)}{A_{0}^{\left(\beta_{2}\right)}(t, z)}$} & \multirow{2}{*}{$\tilde{\zeta}_{1}^{\left(\beta_{2}\right)}(\omega, z)$} & \multirow{2}{*}{$\frac{\tilde{A}_{1}^{\left(\beta_{2}\right)}(\omega, z)}{\tilde{A}_{0}^{\left(\beta_{2}\right)}(\omega, z)}$} \\
\hline & $A_{1}^{\left(\beta_{2}\right)}(t, z)$ & (16) & & & & \\
\hline
\end{tabular}

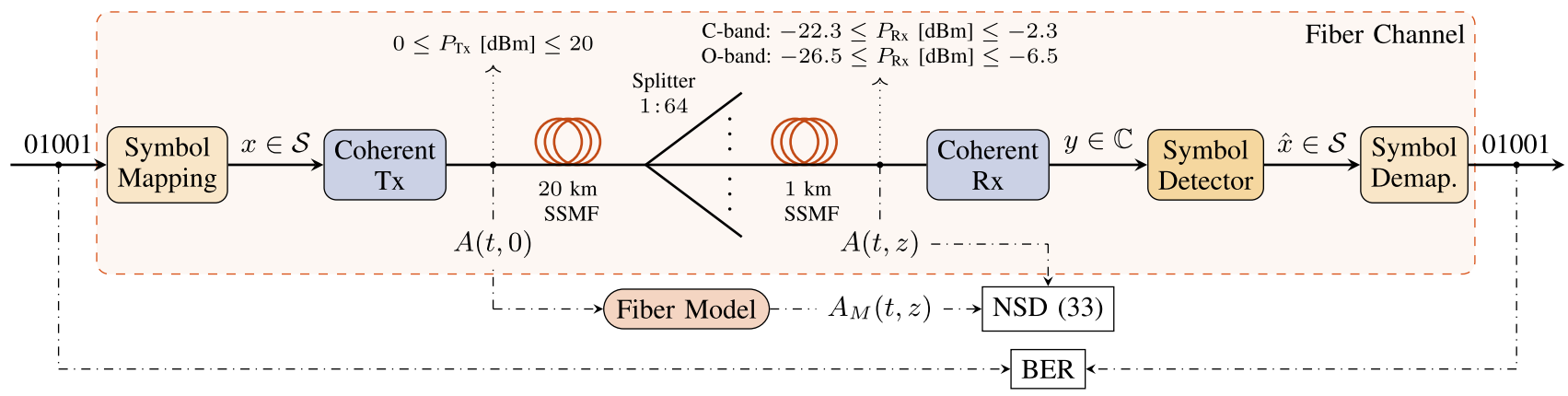

Fig. 2. PON system setup used for the simulations in this paper. This system presents low accumulated dispersion and operates in the highly nonlinear regime for the used range of input powers. The NSD is calculated using the fiber output $A$, obtained via the SSFM, and a fiber model output $A_{M}$. The NSD exact formula given by (37). The BER is estimated using the bits corresponding to the input symbols $x$ and the bits corresponding to the estimated input symbols $\hat{x}$, where $x, \hat{x} \in \mathcal{S}$ and $\mathcal{S}$ is the set of constellation points. Demap.: demapping.

respective model becomes inaccurate. To address this problem in the LP case, [10] proposed to replace $A_{\mathrm{LP}}^{(\theta)}(t, z)$ by $A_{\mathrm{RP}}^{(\theta)}(t, z)$ whenever $\left|A_{0}(t, z)\right|<\epsilon$, where $\epsilon>0$ is a fixed threshold. In our implementation, we chose to replace $A_{\mathrm{LP}}^{(\theta)}(t, z)$ by $A_{\mathrm{RP}}^{(\theta)}(t, z)$ whenever $\left|A_{\mathrm{LP}}^{(\theta)}(t, z)\right|>c\left|A_{\mathrm{RP}}^{(\theta)}(t, z)\right|$ for a certain fixed real $c>$ 0 . Similarly, we replace $\tilde{A}_{\mathrm{FLP}}^{(\theta)}(\omega, z)$ with $\tilde{A}_{\mathrm{RP}}^{(\theta)}(\omega, z)$ whenever $\left|\tilde{A}_{\mathrm{FLP}}^{(\theta)}(\omega, z)\right|>c\left|\tilde{A}_{\mathrm{RP}}^{(\theta)}(\omega, z)\right|$ or $\left|\tilde{A}_{\mathrm{FLP}}^{(\theta)}(\omega, z)\right|=0$, where $\tilde{A}_{\mathrm{RP}}^{(\theta)}$ is the Fourier transform of $A_{\mathrm{RP}}^{(\theta)}$. The value of $c$ for each model was heuristically found and is discussed in Section III-D.

Together with the models in the previous sections, we obtained six perturbation models: two RPs in (3) and (14); two LPs in (30) and (31); and two FLPs in (35) and (36). Table I summarizes these six first-order perturbation methods. As shown in Table I, LP and FLP can be obtained using the RP terms.

\section{Simulation SETUP AND Results}

The model presented in this work is validated in a PON transmission scenario where the accumulated dispersion is expected to be low. Fig. 2 shows the coherent PON system setup under consideration. The fiber parameters are given in Table II. As depicted in the figure, we consider a standard single mode fiber (SSMF) of $20 \mathrm{~km}$, followed by a splitter of ratio 1:64 and a final fiber segment of $1 \mathrm{~km}$. With this split ratio, the total link loss is $22.3 \mathrm{~dB}$ for the $\mathrm{C}$-band and $26.5 \mathrm{~dB}$ for the $\mathrm{O}$-band. The fiber input power $P_{\mathrm{Tx}}$ varies from 0 to $20 \mathrm{dBm}$, which leads to a received power $P_{\mathrm{Rx}}$ between -22.3 and $-2.3 \mathrm{dBm}$ in the C-band and between -26.5 and $-6.5 \mathrm{dBm}$ in the O-band. The
TABLE II

Fiber PARAMETERS FOR C- AND O-BAND TRANSMISSION

\begin{tabular}{|c||c|c|}
\hline Parameter & C-band & O-band \\
\hline Wavelength $\lambda[\mathrm{nm}]$ & 1550 & 1310 \\
$\alpha[\mathrm{dB} / \mathrm{km}]$ & 0.2 & 0.4 \\
$\beta_{2}\left[\mathrm{ps}^{2} / \mathrm{km}\right]$ & -21.67 & -0.2 \\
$\gamma[1 / \mathrm{W} / \mathrm{km}]$ & 1.2 & 1.4 \\
$\beta_{3}\left[\mathrm{ps}^{3} / \mathrm{km}\right]$ & - & 0.0765 \\
\hline
\end{tabular}

range of powers was chosen to cover and go beyond launch powers for typical PON systems according to [20], [21]. All the results were obtained using randomly generated bits which were mapped into symbols drawn from a quadrature phase shift keying (QPSK) constellation $\mathcal{S}=( \pm 1 \pm j) / \sqrt{2}$. The coherent transmitter applies pulse shaping and scales the waveform such that the average transmitted power is $P_{\mathrm{Tx}}$. The coherent receiver undo the waveform scaling, and then applies matched filtering and sampling, without chromatic-dispersion compensation. The symbol rate of the transmitted signal is 10 Gbaud for both Cand O-band systems. The considered pulse shape is a root-raised cosine (RRC), with a roll-off factor of 0.1 . We consider a noiseless scenario since for the considered bandwidth and received powers, nonlinear distortions dominate over the shot noise [19], [22].

In the considered system setup, we want to evaluate the impact of nonlinearities and dispersion on the models. For evaluating the impact of the nonlinearities, the power was varied as specified before. The effect of the dispersion in the models is evaluated by comparing the $\mathrm{C}$-band and the O-band scenarios, which have 


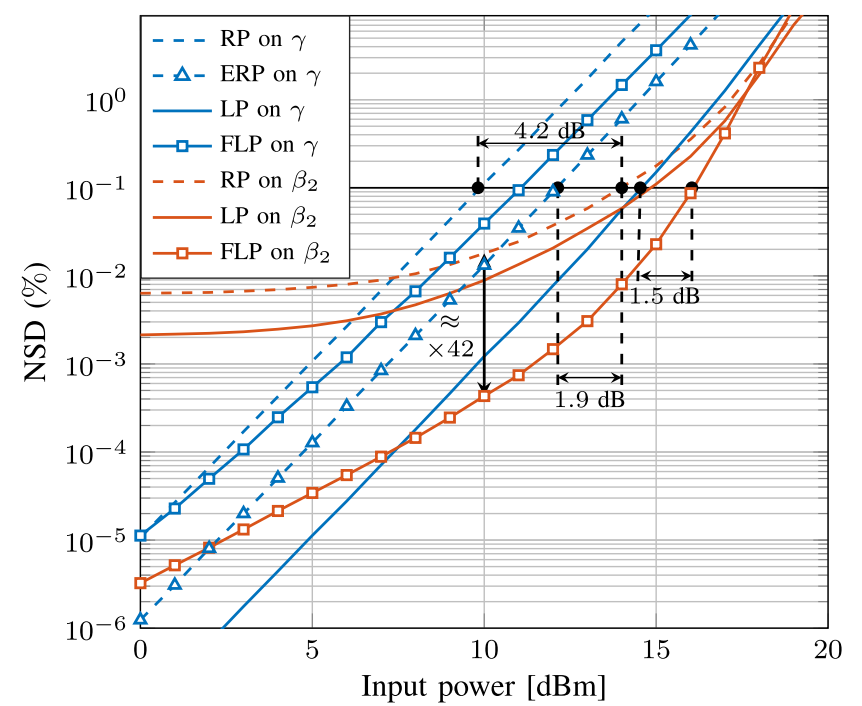

Fig. 3. NSD for for RP, ERP, LP and FLP on $\gamma$ and on $\beta_{2}$ in the C-band using the system in Fig. 2. The fiber parameters are given in Table II.

different $\beta_{2}$ values. We consider the effect of the TOD in the O-band since in that regime values of $\beta_{2}$ are low. Therefore, the equation used for the SSFM simulation in the presence of TOD is (10). In addition, our LP on $\gamma$ results are also accounting for TOD by using (11).

We evaluate the model accuracy on two levels, comparing either the channel output waveforms or detected symbols. For the former, no receiver is considered. The accuracy is quantified using the normalized squared deviation (NSD) metric [5], [11]

$$
\mathrm{NSD} \triangleq \frac{\int_{-\infty}^{\infty}\left|A_{M}(t, z)-A(t, z)\right|^{2} \mathrm{~d} t}{\int_{-\infty}^{\infty}|A(t, z)|^{2} \mathrm{~d} t},
$$

where $A_{M}$ is a model output (i.e., $A_{\mathrm{LP}}^{(\gamma)}$ or $A_{\mathrm{FLP}}^{\left(\beta_{2}\right)}$, for example) and $A$ is the true fiber output obtained from the SSFM. The NSD integrates the absolute error squared over the entire signal duration, and normalizes it with the energy of the signal $A$. Therefore, the lower the NSD, the more accurate is the waveform predicted by the model. The inputs for the NSD calculation are illustrated in Fig. 2.

For the symbol-level evaluation, decision regions are optimized according to each model as described in Section III-B. In that section, the accuracy is quantified in terms of BER (illustrated in Fig. 2), while in Section III-C, the accuracy is quantified in terms of achievable information rate (AIR). Section III-D investigate numerical implementation aspects, such as time complexity and the choice of the threshold $c$.

\section{A. Waveform Comparison}

Fig. 3 shows the NSD for RP, LP, and FLP on $\gamma$ and on $\beta_{2}$. As depicted in Fig. 3, FLP on $\beta_{2}$ (solid red line with squares) is the most accurate at powers higher than $7.5 \mathrm{dBm}$ and NSD below $0.1 \%$, while LP on $\gamma$ (solid blue line) is the most accurate at powers lower than $7.5 \mathrm{dBm}$. FLP on $\gamma$ (solid blue line with squares) and LP on $\beta_{2}$ (solid red line) have a slightly better performance than RP on $\gamma$ (dashed blue line) and RP on $\beta_{2}$

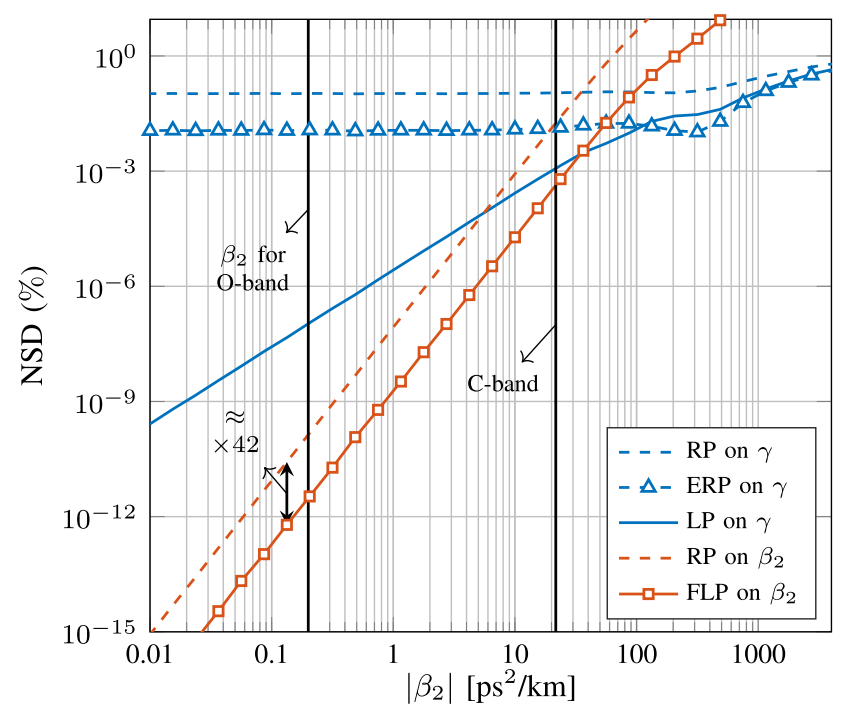

Fig. 4. NSD versus $\left|\beta_{2}\right|$ (negative $\beta_{2}$ ) for five models at an input power of $10 \mathrm{dBm}$. The system is represented in Fig. 2. All considered models except RP on $\gamma$ get higher accuracy as $\left|\beta_{2}\right|$ decreases.

(dashed red line), respectively. However, we do not consider FLP on $\gamma$ and LP on $\beta_{2}$ further in this paper, since their performance is surpassed by LP on $\gamma$ and FLP on $\beta_{2}$, respectively. RP on $\beta_{2}$ crosses the $0.1 \%$ NSD line at an input power approximately $4.2 \mathrm{~dB}$ higher than RP on $\gamma$, at 14 and $9.8 \mathrm{dBm}$, respectively. This gap in favor of RP on $\beta_{2}$ was expected since input powers greater than $10 \mathrm{dBm}$ and small distances such as $20 \mathrm{~km}$ put the fiber in the highly nonlinear regime with low accumulated dispersion. The gap of $4.2 \mathrm{~dB}$ is reduced to $1.9 \mathrm{~dB}$ when comparing ERP on $\gamma$ and RP on $\beta_{2}$. This reduction is justified by the change of variables made in (8), which improves the nonlinear tolerance of that model with respect to RP on $\gamma$. As discussed in [11], RP on $\beta_{2}$ is accurate on this regime, while RP on $\gamma$ loses accuracy at high powers. If we change from RP on $\gamma$ to LP on $\gamma$, the latter outperforms RP on $\beta_{2}$ for powers below $16 \mathrm{dBm}$. This gain in accuracy by changing from the LP on $\gamma$ to the RP on $\gamma$ was previously shown in [10]. In addition, LP on $\gamma$ has its performance increased due to low accumulated dispersion. By letting $\beta_{2} \rightarrow 0$ in (30), LP on $\gamma$ tends to the NLPN solution, which is accurate in very low dispersion scenarios [11].

The dependence of the models on $\left|\beta_{2}\right|$ can be seen in Fig. 4, where four models are compared at a fixed power of $10 \mathrm{dBm}$ for different values of $\left|\beta_{2}\right|$ and the other parameters for C-band transmission (with no TOD). All the simulated $\beta_{2}$ values were negative. Among the four models, RP and ERP on $\gamma$ are the only ones that are virtually invariant to changes in $\beta_{2}$ for $\left|\beta_{2}\right|<30$ $\mathrm{ps}^{2} / \mathrm{km}$. Nevertheless, RP on $\gamma$ has worse accuracy than LP on $\gamma$ for all displayed values of $\beta_{2}$. When increasing $\left|\beta_{2}\right|$, LP on $\gamma$ increases its NSD at a rate of approximately $10^{2}$ per decade. Although LP on $\gamma$ outperforms RP on $\beta_{2}$ at $10 \mathrm{dBm}$ for the C-band, the latter has an increasing rate of approximately $10^{4}$ per decade, and surpasses the accuracy of LP on $\gamma$ for $\beta_{2}$ values lower than $-6 \mathrm{ps}^{2} / \mathrm{km}$. If we now also consider FLP on $\beta_{2}$, we can gain approximately 42 times in NSD accuracy with respect to RP on $\beta_{2}$ at $10 \mathrm{dBm}$. This gap can also be seen in Fig. 3 


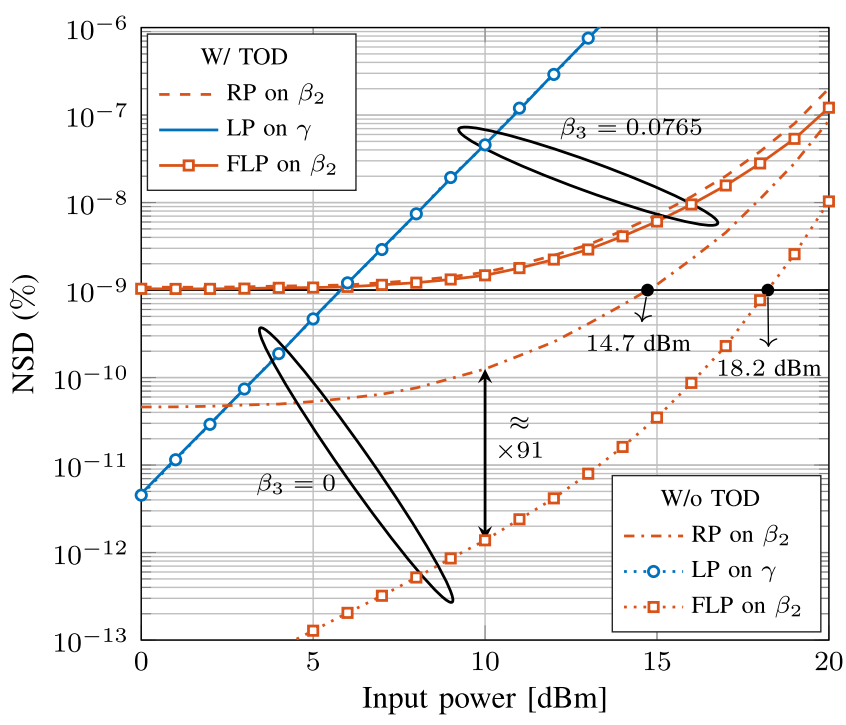

Fig. 5. NSD for the four models in the O-band for the system in Fig. 2. Results without TOD are also shown.

and remains approximately constant for different values of $\beta_{2}$, since both RP and FLP on $\beta_{2}$ have the same increasing rate of approximately $10^{4}$ per decade. The higher increasing rate for RP and FLP on $\beta_{2}$ when compared with LP on $\gamma$ shows that the two former models converge to the true solution of (1) faster than the latter model when decreasing the accumulated dispersion. For $\beta_{2}=-21.67$ (C-band), FLP on $\beta_{2}$ already outperforms LP on $\gamma$. In addition, we see back in Fig. 3 that FLP on $\beta_{2}$ crosses the line for an NSD of $0.1 \%$ at an input power $1.5 \mathrm{~dB}$ higher than LP on $\gamma$. For high values of $\left|\beta_{2}\right|>30 \mathrm{ps}^{2} / \mathrm{km}$, the NSD for LP and for ERP on $\gamma$ start to converge to a value closer to the NSD for RP on $\gamma$.

The results for LP on $\gamma$, RP on $\beta_{2}$, and FLP on $\beta_{2}$ using the O-band parameters in Table II are shown in Fig. 5, where the NSD is displayed as a function of the input power. First, we consider a system without TOD (dotted and dashed dotted lines). As shown in Fig. 5, the NSD for LP on $\gamma$ and FLP on $\beta_{2}$ significantly decay when reducing the input power in the absence of TOD. On the other hand, RP on $\beta_{2}$ converges to an NSD of approximately $4.6 \cdot 10^{-11 \%}$ for powers lower than $2 \mathrm{dBm}$. This convergence to a non-zero NSD value reflects the mismatch between (13) and (14) in the absence of nonlinearities. FLP on $\beta_{2}$ outperforms LP on $\gamma$ for all the displayed input powers, while $\mathrm{RP}$ on $\beta_{2}$ outperforms LP on $\gamma$ for input powers higher than $2.5 \mathrm{dBm}$. At $10 \mathrm{dBm}$, the difference in NSD between RP and FLP on $\beta_{2}$ is approximately 91 times, which is different from the 42 factor for the $\mathrm{C}$-band results at the same input power (see Fig. 4). This discrepancy is due to the new set of $\gamma$ and $\alpha$ values, which boost the difference between the two models at that input power.

The results for the O-band in Fig. 5 shows that when TOD is considered, RP and FLP on $\beta_{2}$ converge to a constant NSD of $10^{-9}$ for input powers lower than $3 \mathrm{dBm}$. This behavior can be explained by the absence of TOD in the model derivations. The error introduced by not accounting for TOD becomes approximately constant when input powers are lower than $3 \mathrm{dBm}$, and dominates the error introduced by incorrectly modeling the other fiber effects. From 0 to $20 \mathrm{dBm}$, the performance of RP and FLP on $\beta_{2}$ in the system with TOD is worse than without TOD. This behavior is expected, since for the system without TOD, RP and FLP on $\beta_{2}$ cross the constant NSD of $10^{-9}$ (TOD error floor for low powers) only at $14.7 \mathrm{dBm}$ and $18.2 \mathrm{dBm}$, respectively. For LP on $\gamma$, the NSD for the system without TOD is virtually the same as for the system with TOD since we already account for TOD in the model derivation, as done in (11).

\section{B. Decision Region Optimization}

As discussed in [11], comparing models in discrete time can lead to slightly different conclusions than on a waveform level. For this reason, this section compares LP on $\gamma$, FLP on $\beta_{2}$, and SSFM results at the symbol level, measured by BER. The results are shown for the C-band system with parameters given in Table II. To obtain the received symbols, the output waveform from these three models is filtered by a matched filter and sampled as done for the signal $A(\cdot, z)$ in Fig. 2 . We do not include chromatic dispersion compensation (CDC) at the receiver due to the high amount of interaction between chromatic dispersion and nonlinearities which cannot be efficiently compensated by CDC. For example, the BER for this setup with a CDC block is virtually the same as the BER without CDC when using a minimum distance symbol detector. The resulting complex samples are used to optimize decision regions for each model, originating a symbol detector. Finally, SSFM simulations are performed to validate the accuracy of each symbol detector when receiving the true (SSFM) output waveform.

The decision region optimization for the symbol detector is based on the histogram of the received complex samples. This histogram-based (HB) detector follows the principle of choosing the most probable transmitted symbol, given that the corresponding received sample falls in certain area of the complex plane. This principle corresponds to the maximum a-posteriori (MAP) rule

$$
\hat{x}=\operatorname{argmax}_{s_{m} \in \mathcal{S}} \operatorname{Pr}\left\{X=s_{m} \mid Y=y\right\} .
$$

where $X$ and $Y$ are random variables associated with the transmitted symbols and received samples (see Fig. 2), respectively, and $s_{m} \in \mathcal{S}$ where $\mathcal{S}=\left\{s_{1}, \ldots, s_{M}\right\}$ is the set of constellation points with cardinality $M$ and $1 \leq m \leq M$. The MAP rule, as stated in (38), is optimal for a memoryless channel, and thus, suboptimal for the optical fiber channel, which includes memory.

To build the HB detector and numerically approximate the rule in (38), training symbols are transmitted through the fiber in order to obtain an estimation of the probability distribution. Their respective received samples will fall in a specific bin, which is an small area in the complex plane. For each bin, we count the number $n_{m, b}$ of received samples that fall inside the $b$-th bin and were drawn from the $m$-th constellation point, where $1 \leq b \leq B, 1 \leq m \leq M$, and $B$ is the total number of bins. The value of $m=\hat{m}_{b}$ that maximizes $n_{m, b}$ is considered to be the most probable transmitted constellation point for that 


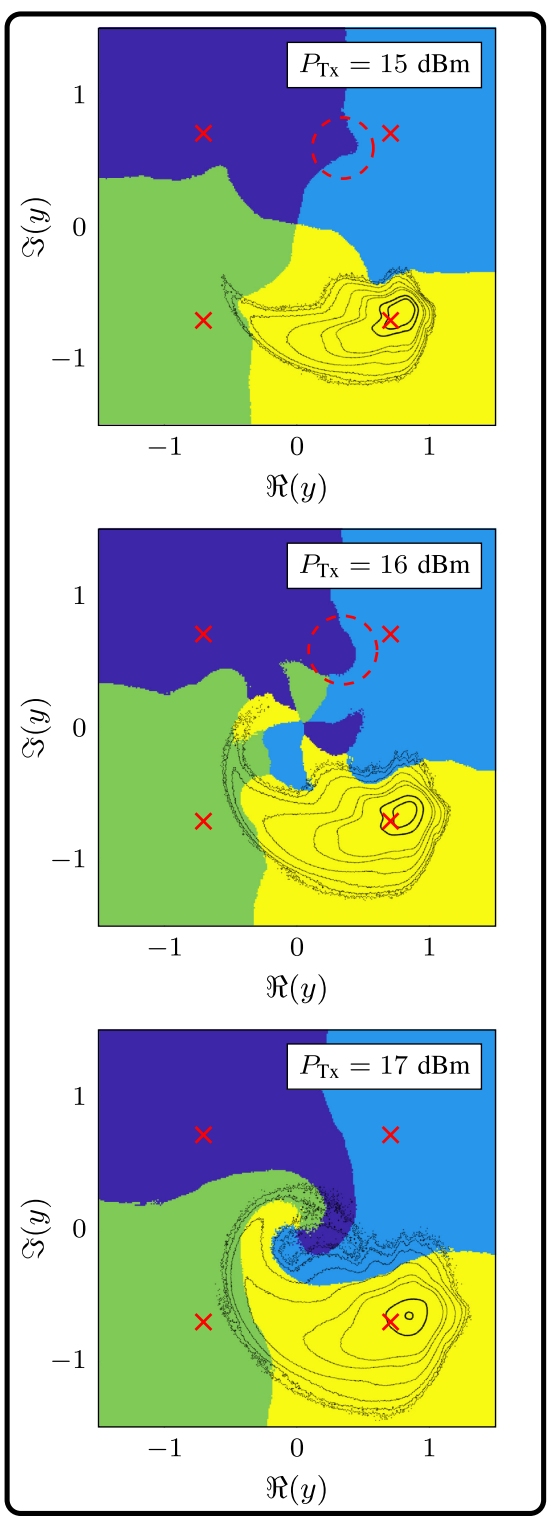

(a) SSFM
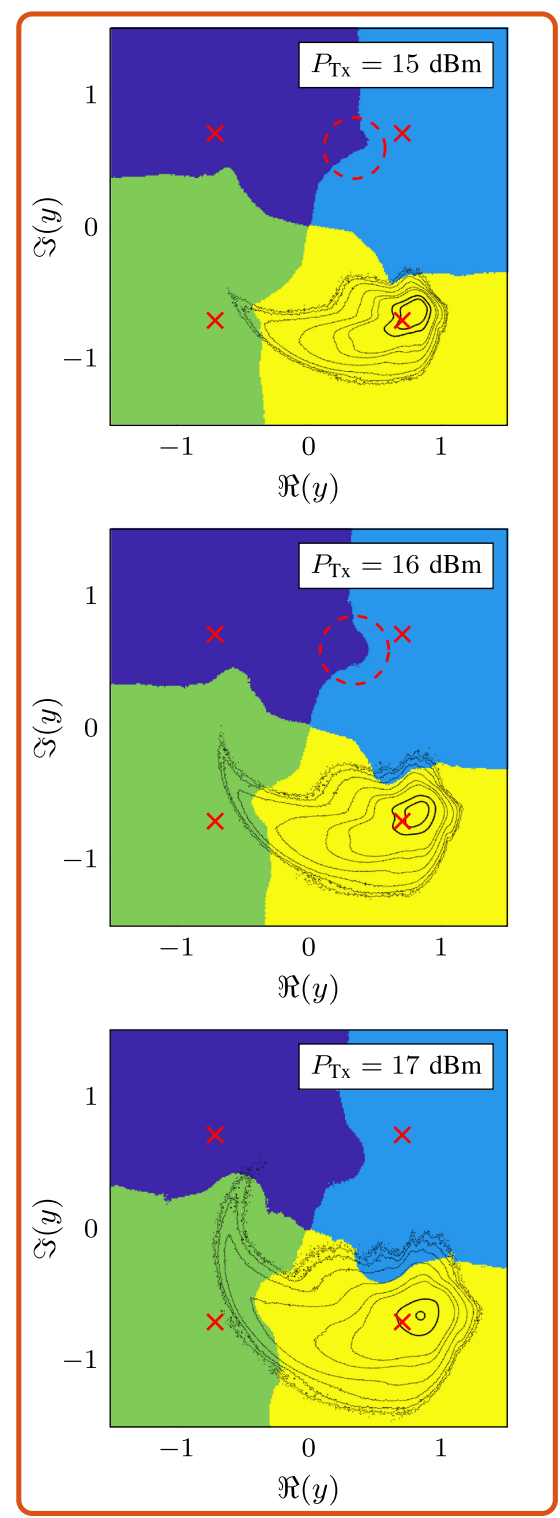

(b) FLP on $\beta_{2}$.
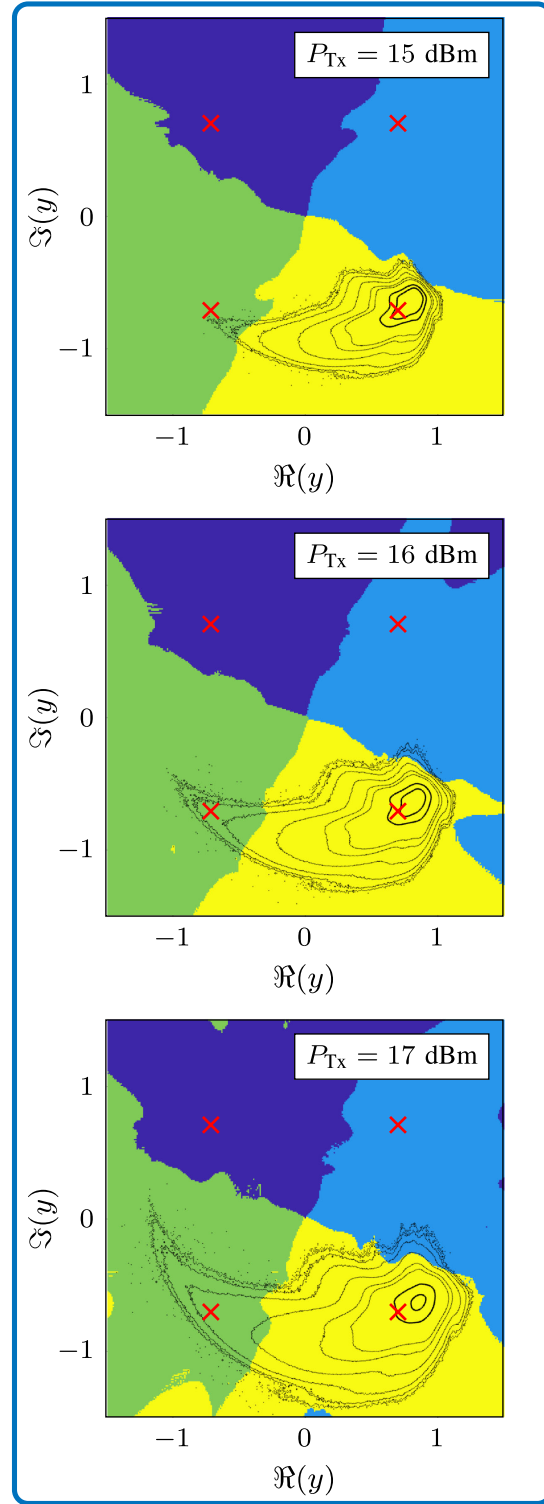

(c) LP on $\gamma$.

Fig. 6. Optimized decision regions obtained with $\mathrm{HB}$ detection after the propagation for $P_{\mathrm{Tx}} \in\{15,16,17\}$ dBm of a QPSK constellation based on: (a) SSFM; (b) FLP on $\beta_{2}$; (c) LP on $\gamma$. Each of the four differently colored areas represents the decision region for a specific constellation point. The respective constellation points are illustrated with red crosses. A contour plot of the histogram for the received samples for the transmitted symbol $(1-j) / \sqrt{2}$ is plotted on top of each subfigure.

bin. If $\max _{m}\left\{n_{m, b}\right\}=0$, we replace $b$ by the closest bin $b^{\prime}$ in Euclidean distance such that $\max _{m}\left\{n_{m, b^{\prime}}\right\}>0$, and decide $\hat{m}_{b}=\operatorname{argmax}_{m}\left\{n_{m, b^{\prime}}\right\}$. After obtaining $\hat{m}_{b}$ for all possible $b$, the decision regions are defined and every time a received sample $y$ is received in the $b$-th bin, we assume that received symbol $\hat{x}=$ $s_{\hat{m}_{b}}$ was transmitted. For computational reasons, we only consider a subset $\mathcal{A}=\{z \in \mathbb{C}:|\Re\{z\}|<2,|\Im\{z\}|<2\}$ of the complex plane, divided into $B=400 \times 400$ square bins of side 0.01 . This region is sufficient to contain virtually all the received samples in the simulation. We simulate 2000 times the transmission of $2^{18}$ symbols, totalling approximately $5.2 \cdot 10^{8}$ symbols to obtain histograms that define $n_{m, b}$. Each sequence of $2^{18} \mathrm{sym}-$ bols was randomly generated and the transmitted waveform had an oversampling factor of 16 samples per symbol. The decision regions were optimized for each transmitted power separately.

Fig. 6 depicts the decision regions obtained by using HB detectors for SSFM, FLP on $\beta_{2}$, and LP on $\gamma$ at input powers $P_{\mathrm{Tx}} \in\{15,16,17\} \mathrm{dBm}$. The transmitted constellation is illustrated with red crosses. Fig. 6 also includes contour plots of the histogram for the received samples when transmitting the constellation point $(1-j) / \sqrt{2}$. The decision regions associated to this constellation point are represented in yellow and contain most of the received samples shown by the contour plots. The samples that fall outside the yellow regions are not classified as $(1-j) / \sqrt{2}$ since there are more received samples originating from another constellation point in those specific bins. 


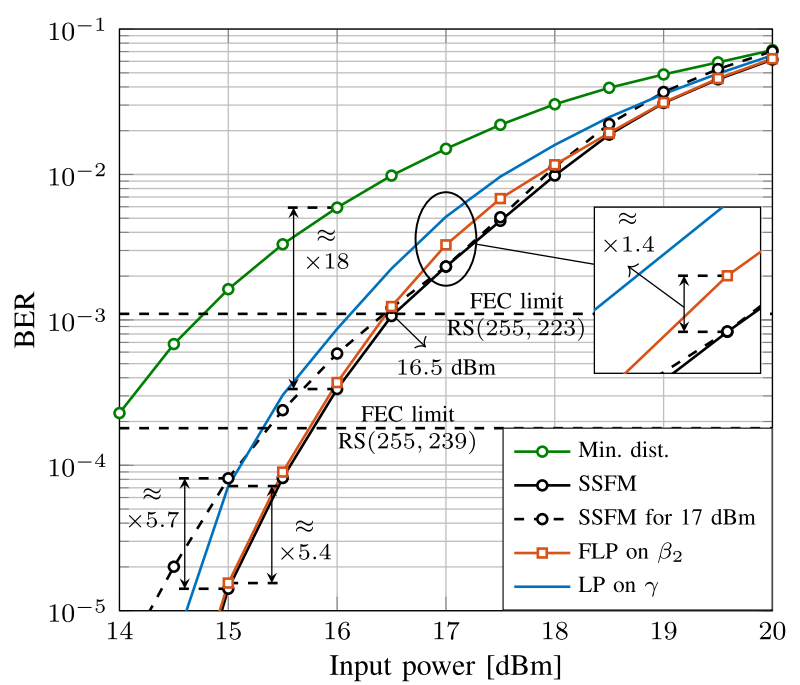

Fig. 7. BER versus input power for receivers using $\mathrm{HB}$ detectors. The HB detectors were obtained via SSFM, LP on $\gamma$, and FLP on $\beta_{2}$. In all cases, the channel was simulated in the C-band using the SSFM. BER results for fixing the SSFM detector obtained for $17 \mathrm{dBm}$ input power are also presented. Min. dist.: Minimum distance.

The optimum decision regions are considered as the ones obtained via SSFM in Fig. 6(a). At $15 \mathrm{dBm}$, the decision regions differ significantly from the four quadrants of the complex plane that represent the minimum distance decision regions. This difference originates from the nonlinearities, which creates a non-Gaussian distribution of the received samples. Due to the high nonlinear effect present at $17 \mathrm{dBm}$, the SSFM decision regions and the contour plots follow a spiral shape. The decision regions obtained by FLP on $\beta_{2}$ are shown in Fig. 6(b). At $15 \mathrm{dBm}$, these decision regions are similar to the SSFM ones for the same $P_{\mathrm{Tx}}$. However, at $17 \mathrm{dBm}$, these regions do not present a spiral shape, as in Fig. 6(a). This new behavior can be explained by the contour plots. The curvature of the contour plots in Fig. 6(b) is lower than the curvature in Fig. 6(a), which results in the symbols from the tail of the contour plot falling in bins with a high number of points transmitted from another constellation point. The curvature for the contour plot of symbols obtained via LP on $\gamma$ in Fig. 6(c) is even lower than for FLP on $\beta_{2}$ in Fig. 6(b). This fact results in decision regions with less accentuated curves in center of Fig. 6(c) for each $P_{\mathrm{Tx}}$. At $15 \mathrm{dBm}$, the LP on $\gamma$ decision regions already differ significantly from the SSFM ones. For example, at both 15 and $16 \mathrm{dBm}$, a small lobe (red dashed circle) present in both SSFM and FLP on $\beta_{2}$ decision regions is not present in the LP on $\gamma$ ones. The shape of the contour plots indicate that the decision regions obtained with FLP on $\beta_{2}$ might perform closer to the SSFM decision regions than the ones obtained with LP on $\gamma$, as will be discussed next.

The HB detectors obtained using LP on $\gamma$, FLP on $\beta_{2}$, and SSFM are compared in a system whose fiber propagation is modeled by the SSFM. The results are shown in Fig. 7, where the BER is evaluated for different launch powers using the obtained HB detectors. The latter are obtained using the SSFM for fiber propagation. The BER was estimated after averaging the results for 100 realizations of randomly generated sequences of $2^{17}$ symbols, totalling approximately $1.3 \cdot 10^{7}$ symbols. As shown in Fig. 7, the SSFM HB detector (black curve) shows the lowest BER for the displayed input powers. The minimum distance HB detector gives the worst performance since it assumes a Gaussian distribution of the received samples. This detector is obtained by minimum Euclidean distance from the received samples to the possible transmitted symbols. Replacing the minimum distance $\mathrm{HB}$ detector by the SSFM one at $16 \mathrm{dBm}$ reduces the BER approximately 18 times (from $5.9 \cdot 10^{-3}$ to $3.3 \cdot 10^{-4}$ ). At $15 \mathrm{dBm}$, the BER for FLP on $\beta_{2}$ is 4.6 times lower than for LP on $\gamma$. As expected, results for FLP on $\beta_{2}$ are closer to the SSFM results than LP on $\gamma$. The highest gap between the SSFM and FLP on $\beta_{2}$ occurs at $17 \mathrm{dBm}$, where the BER for FLP on $\beta_{2}$ is 1.4 times higher than the BER for SSFM. The fact that a higher gap appears from $16.5 \mathrm{dBm}$ could be related to the high NSD observed in Fig. 3 for FLP on $\beta_{2}$ at that range of input powers. Fig. 7 also shows the performance of the SSFM detector obtained at $17 \mathrm{dBm}$ when used for other input powers. As the input power distance from $17 \mathrm{dBm}$ increases, the performance of the SSFM detector for $17 \mathrm{dBm}$ decreases with respect to the performance of other SSFM detectors obtained in those respective input powers. For example, at $15 \mathrm{dBm}$, the BER for the $17 \mathrm{dBm}$ SSFM detector is 5.7 times higher than the $15 \mathrm{dBm}$ SSFM one, matching the performance of LP on $\gamma$. Nevertheless, the BER of the $17 \mathrm{dBm}$ SSFM detector is still lower than the BER for minimum distance detection.

\section{Achievable Information Rates}

FEC is present in modern PON systems to improve system performance [23]. In Fig. 7, pre-FEC BER thresholds are shown for two Reed-Solomon (RS) codes [24]. The considered codes in Fig. 7 are $\operatorname{RS}(n, k)$ with $k=239223$ and $n=255$, where $k$ and $n$ are the information and codeword lengths, resp. These two codes are typical low-complexity RS codes used in PONs [23], [25] and have a code rate of 0.93 and 0.87 , respectively.

Along with RS codes, also stronger FEC codes such as low-density parity-check codes or staircase codes have been proposed for PONs in the literature [26]-[28]. In this section, we evaluate the models for PON systems with hard-decision (HD) FEC in terms of achievable information rates (AIRs) [29]. We consider the simple RS codes described above and a theoretical HD limit for the AIR. The latter is close to the performance of strong HD FEC codes [30, Fig. 8]. The AIRs are obtained by closed-form expressions based on pre-FEC BER.

We consider a family of $\mathrm{RS}$ codes $\operatorname{RS}(n, k)$ with multiple coding rates, where $k$ is varied to obtain different code rates. We use a fixed codeword length ( $n=255$ symbols) in order to constrain the code complexity. For every launch power, $k$ is determined by finding the highest $k$ such that the post-FEC BER falls below a certain threshold. Following [20, Table IV.2], we use $10^{-12}$ as post-FEC BER threshold.

The post-FEC BER $p_{\text {pos }}$ can be approximated by substituting the pre-FEC BER $p$ from the system in Fig. 2 in the analytical expression for binary symmetric channels (BSCs) and boundeddistance decoders [31]

$$
p_{\mathrm{pos}} \approx \frac{1}{n} \sum_{r=t+1}^{n}\left(\frac{p}{p_{s}} r+\frac{1}{2(t-1) !}\right)\left(\begin{array}{l}
n \\
r
\end{array}\right) p_{s}^{r}\left(1-p_{s}\right)^{n-r}
$$




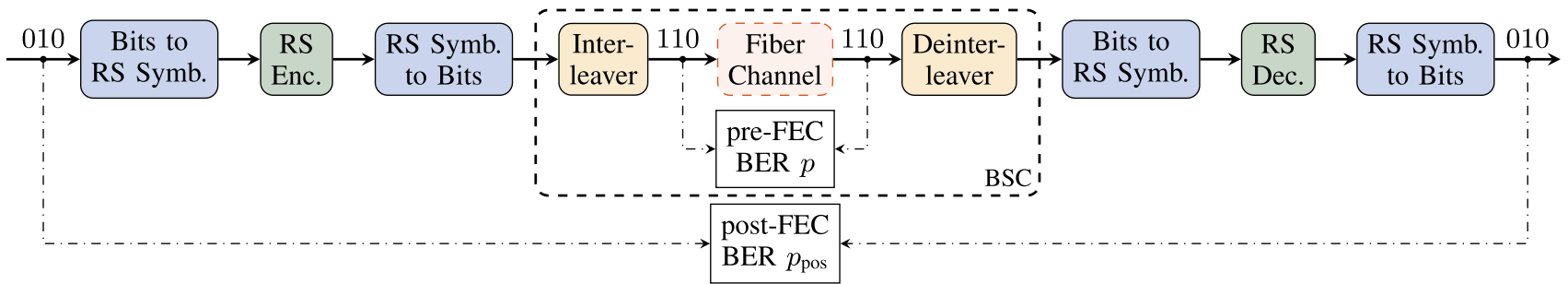

Fig. 8. Encoding/decoding procedures used in this paper for RS codes. The fiber channel block include all components from Fig. 2. The post-FEC BER $p_{\text {pos }}$ is estimated from the pre-FEC BER $p$ using (39). Enc.: encoder; Dec.: decoder; BSC: binary symmetric channel.

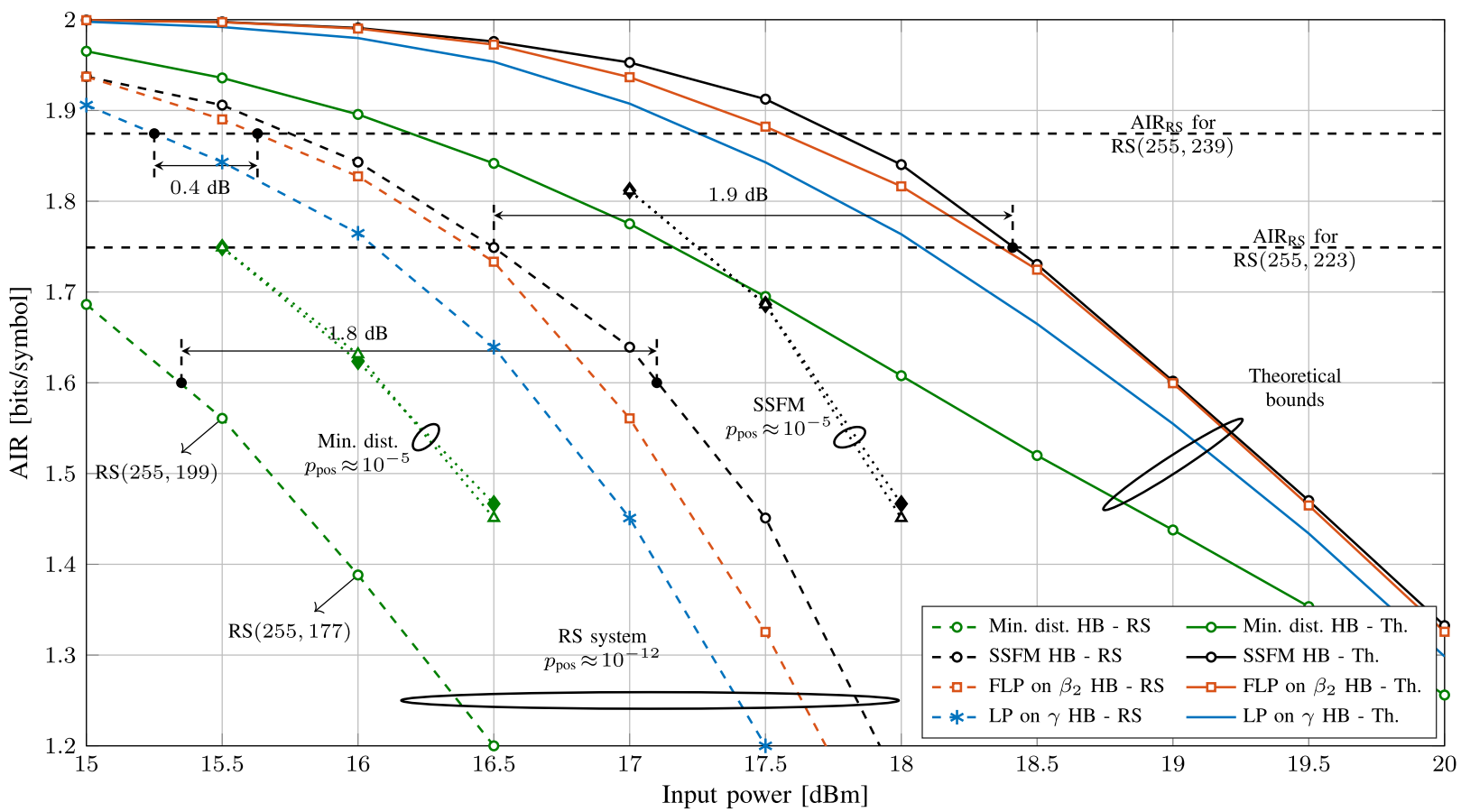

Fig. 9. Comparison of AIRs when using the decision regions obtained in Section III-B. The AIRs were obtained using (40) and (39) at $p_{\text {pos }} \approx 10^{-12}$ for the RS system and (41) for the theoretical bound. The dotted lines represent the comparison between $\mathrm{AIR}_{\mathrm{RS}}$ when approximating $p_{\mathrm{pos}}$ by (39) (white triangles) and AIR $\mathrm{RS}$ when simulating the system in Fig. 8 (filled diamonds), both for $p_{\text {pos }} \approx 10^{-5}$. Th.: theoretical.

where $t=\lfloor(n-k) / 2\rfloor$ is the RS error-correction capability, $p_{s}=1-(1-p)^{m}$ is the (RS) symbol error probability, and $m=\left\lceil\log _{2}(n+1)\right\rceil$ is the number of bits per symbol. To improve the total computation time, a binary search algorithm on $k$ over all integers between 1 and 253 is performed. After finding $k$, the AIR for the RS system ( $\left.\mathrm{AIR}_{\mathrm{RS}}\right)$ is determined by

$$
\mathrm{AIR}_{\mathrm{RS}}=\log _{2}(M) \frac{k}{n}=\frac{2}{255} k,
$$

since $M=4$ for QPSK. We call (40) an achievable information rate since we assume that $p_{\text {pos }}<10^{-12}$ can be considered virtually error-free for the system considered in this paper.

Since the expression in (39) is valid for BSCs, we need to modify the system in Fig. 2 to fulfill that property. The fiber channel in Fig. 2 presents memory due to the interaction of dispersion and nonlinearities. Therefore, in our simulations we included a bit interleaver and a bit deinterleaver so that the fiber channel in Fig. 2 is well-approximated by a BSC. The resulting system, together with the RS encoding and decoding blocks, can be seen in Fig. 8. The system in Fig. 8 was only simulated for $p_{\text {pos }} \approx 10^{-5}$ and was used to validate the results of (39). After this validation, (39) was used instead of simulating the system in Fig. 8.

Fig. 9 depicts the AIRs for the RS system using (39). Before analyzing the results for $p_{\mathrm{pos}} \approx 10^{-12}$, we validate (39) at $p_{\text {pos }} \approx 10^{-5}$ to determine if the system in Fig. 8 can be well-approximated by a BSC. This validation is done assuming a minimum distance detector and is shown by the dotted lines in Fig. 9. The results for the RS simulations of the exact system in Fig. 8 at $p_{\text {pos }} \approx 10^{-5}$ are shown with diamonds. An almost perfect overlap with the results from (39) with the same $p_{\text {pos }}$ (shown with triangles) is observed. The agreement between these curves suggest that the system in Fig. 8 can be approximated by a BSC. Therefore, from now on only (39) is used for the RS systems at $p_{\text {pos }} \approx 10^{-12}$.

We start by comparing the dashed lines in Fig. 9, which represent the AIR RS results in (40). As shown in Fig. 9, using the decision regions obtained by SSFM in a RS system can provide a gain of approximately $1.8 \mathrm{~dB}$ for a rate of $1.6 \mathrm{bits} / \mathrm{symbol}$ in terms of nonlinear tolerance. The crossing point between the rate 
of $\operatorname{RS}(255,223)$ with $\mathrm{AIR}_{\mathrm{RS}}$ for the SSFM decision regions is at $16.5 \mathrm{dBm}$, which closely matches with the crossing point with BER in Fig. 7. When comparing the models, the histogram-based detector obtained using FLP on $\beta_{2}$ outperforms the LP on $\gamma$ one throughout the considered power range, analogously to Fig. 7. Specifically for $\operatorname{RS}(255,239)$, FLP on $\beta_{2}$ outperforms LP on $\gamma$ by approximately $0.4 \mathrm{~dB}$.

The $\mathrm{AIR}_{\mathrm{RS}}$ is also compared with a theoretical bound on harddecision bit-wise AIRs for independent, identically distributed bit errors. The theoretical AIR ( $\mathrm{AIR}_{\mathrm{TH}}$ ) used in this paper is defined as [32]

$$
\operatorname{AIR}_{\mathrm{TH}}=\log _{2}(M)\left(1-\mathrm{H}_{\mathrm{b}}(p)\right),
$$

where $\mathrm{H}_{\mathrm{b}}(p)=-p \log _{2}(p)-(1-p) \log _{2}(1-p)$ is the binary entropy function for the given pre-FEC BER $p$ in the system of Fig. 2. The AIR ${ }_{T H}$ from (41) can be approached by strong FEC codes such as staircase codes, as reported in [30, Fig. 8].

As shown in Fig. 9, the theoretical bounds (solid lines) from (41) show significant gains over the $\mathrm{AIR}_{\mathrm{RS}}$ results. These gains show that, by using codes more complex than $\mathrm{RS}(255, k)$, higher code rates can be achieved or the input power can be improved for a specific rate. For the same code rate as in $\operatorname{RS}(255,223)$, the input power for the theoretical bound on the SSFM decision regions is approximately $1.9 \mathrm{~dB}$ higher than the one for the RS system. However, the complexity and latency of codes that perform close to the theoretical bound should be carefully analysed for a PON system design. The results for the theoretical bounds in in Fig. 9 also show that the histogram-based detector based on the FLP on $\beta_{2}$ outperforms the one based on LP on $\gamma$.

\section{Numerical Analysis}

As mentioned in Section II-D, LP on $\gamma$ and FLP on $\beta_{2}$ suffer from a numerical issue related to the ratio between the first and zeroth RP order. In this section, this issue is further investigated, more details on the models' implementation are presented, and the time-complexity tradeoff is discussed.

The time derivatives present in the $\beta_{2}$ perturbation models were calculated in the frequency domain by using fast Fourier transforms. Once the terms $A_{0}^{\left(\beta_{2}\right)}$ and $A_{1}^{\left(\beta_{2}\right)}$ were obtained, they were used to build both RP and FLP on $\beta_{2}$ models to avoid the recalculation of those terms for FLP. This last trick was analogously done for RP and LP on $\gamma$. To obtain the models RP and LP on $\gamma$, the integral in (5) was calculated using Gauss-Legendre quadrature [33, Ch. 5], [34]. The minimum number of necessary quadrature points to achieve the NSDs displayed for the C-band was only 2 , while for the $\mathrm{O}$-band this number increased to 4 . The weights for the Gauss-Legendre quadrature were obtained from a look-up table. The SSFM calculations previously presented in the paper were performed using uniform (constant) spacial step sizes of $0.1 \mathrm{~km}$. However, for the time complexity comparison in this section, we used logarithmic step sizes to increase the accuracy for a given number of steps [35]. A multiplicative correction factor of 0.6 was used to adjust $\alpha$ in the logarithmic step size calculation. This correction factor changes the distribution of the step sizes in order to improve the few-steps SSFM NSD. The simulations were performed on a NVIDIA ${ }^{\circledR}$ Tesla $^{\circledR}{ }^{\circledR} \mathrm{P} 100$ GPU and the code written in MATLAB ${ }^{\circledR}$. The code used for

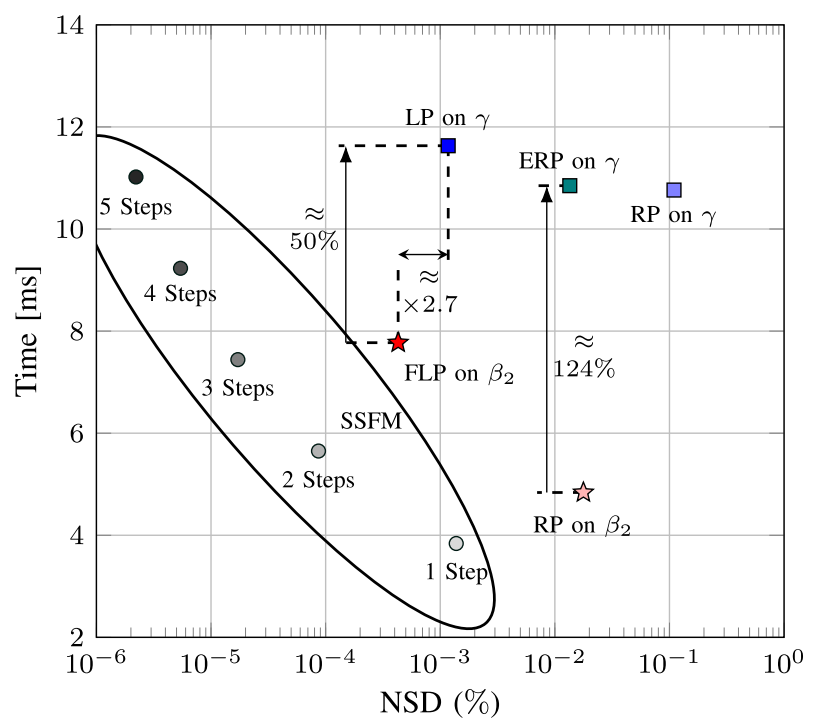

Fig. 10. Average simulation time for most of the models considered in this paper. The simulated system is a $20 \mathrm{~km}$ fiber in C-band with input power $10 \mathrm{dBm}$. The code used to generate the models can be found on [36]. Few-steps SSFM simulations are also added to the figure (circles).

the models can be found in [36]. The simulation setup in this section differs slightly from the one considered in Section III-A. We consider C-band simulations at a fixed $10 \mathrm{dBm}$ input power. The splitter and the additional $1 \mathrm{~km}$ fiber are ignored in order to measure the time and accuracy of a single fiber segment for each method.

Fig. 10 shows the simulation time versus NSD for most of the models considered on this paper. The NSD values were averaged over 100 simulations, while the simulation time was averaged over 3000 realizations of the MATLAB function gputimeit $^{\circ}$ for each model. In each simulation, $2^{17}$ symbols were transmitted, with an oversampling factor of 16 . Random symbol sequences were generated when obtaining each NSD sample, while the input waveform for the simulation time measurements was fixed for all models. The NSD for the models and for SSFM simulations with a small number of spatial steps was calculated using as the reference waveform SSFM simulations with 70 logarithmically spaced steps. These steps were calculated using the symmetrized SSFM approach [1], where the nonlinear effect is included in the middle of each step. The few-steps SSFM simulations also used symmetrized logarithmically spaced steps. As depicted in Fig. 10, 2 symmetric steps SSFM simulations already yield higher accuracy in lower simulation time than all the other models in that figure for this system, except for RP on $\beta_{2}$ which has a slightly lower simulation time. Therefore, one might prefer using few-steps SSFM for numerical simulations instead of analytical models for this system.

However, our motivation for developing new integral-form models is to focus on better understanding fiber propagation instead of numerical aspects. Analogously to RP on $\gamma$, we believe that the $\beta_{2}$ perturbation models could also be used to build low-complexity receivers and performance prediction formulas. When comparing analytical models, Fig. 10 shows that changing from RP to ERP on $\gamma$ requires virtually no increase in time, while yielding a lower NSD. The NSD of ERP on $\gamma$ is comparable to 


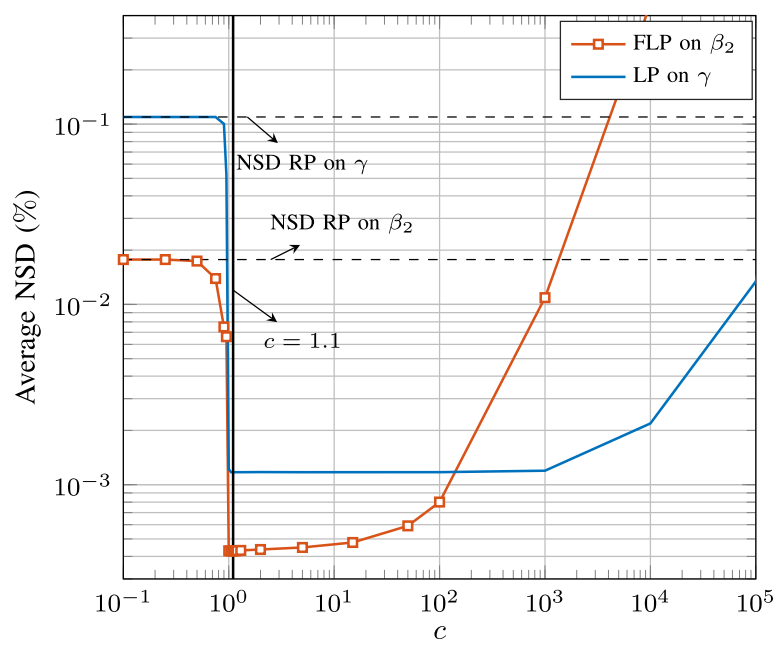

Fig. 11. Average NSD versus threshold $c$ over 500 simulations for LP on $\gamma$ and FLP on $\beta_{2}$. For $c=1.1$, the average NSD is approximately the minimum for both models.

the NSD of RP on $\beta_{2}$ at that input power. Nevertheless, ERP on $\gamma$ requires approximately $124 \%$ more simulation time than RP on $\beta_{2}$. The gap in time between RP and FLP on $\beta_{2}$ is higher than the gap between RP and LP on $\gamma$. This difference might be explained by the additional fast Fourier transforms required in the FLP method and by the additional search $\tilde{A}_{0}^{\left(\beta_{2}\right)}(\omega, z)=0$, as stated in Section II-D. Although having a lower gap from RP on $\gamma$, the simulation time for LP on $\gamma$ is $50 \%$ higher than for FLP on $\beta_{2}$. In addition, FLP on $\beta_{2}$ presents approximately 2.7 times lower NSD than LP on $\gamma$.

The choice of the threshold $c$ used for both LP and FLP methods is also investigated. Fig. 11 illustrates the average NSD versus $c$ over 500 simulations. As shown in Fig. 11, for $c<0.02$, the NSD of LP on $\gamma$ and FLP on $\beta_{2}$ converge to the NSD of their respective RP models. For $c>10$, the NSD of FLP on $\beta_{2}$ starts to increase significantly. This increase is due to high energy isolated points where the value of the zeroth order perturbation term is significantly smaller than the first order one. The lower the value of $c$, the more these isolated points are filtered. The same behavior happens for LP on $\gamma$ for $c>10^{3}$. At $c=1.1$, the NSD is virtually the minimum for both models and was the chosen threshold for simulations.

\section{CONCLUSIONS}

In this paper we presented a novel model for optical fiber transmission and evaluated its performance for a passive optical network system. The proposed model was derived as an improved version of the regular perturbation on the GVD parameter model. The improvement was obtained by applying frequency logarithmic perturbation on the GVD parameter. Both regular and frequency logarithmic perturbation on the GVD parameter models are suitable in the weakly dispersive and highly nonlinear regime, whereas the frequency logarithmic perturbation is able to surpass the limitations of the regular perturbation.

Apart from the regular perturbation on the GVD parameter, the proposed model was compared with two other models present in the literature: regular and logarithmic perturbation on the Kerr nonlinear coefficient. For a fixed normalized squared deviation of $0.1 \%$, the proposed model was accurate at $1.5 \mathrm{~dB}$ higher input powers compared to logarithmic perturbation on the Kerr nonlinear coefficient. Both frequency logarithmic and regular perturbation on the GVD parameter exhibit the highest convergence rate to the split-step Fourier method results when reducing the dispersion effect. The proposed model also proved to be more suitable for symbol and bit detection, with and without FEC.

Possible extensions of this work are higher-order logarithmic perturbation models, perturbation on the GVD parameter for dual-polarization systems, and nonlinearity-compensation techniques based on the proposed model.

\section{ACKNOWLEDGMENT}

The authors would like to thank Dr. Tobias Fehenberger (ADVA Optical Networking) for fruitful discussions about the regular perturbation on $\gamma$. We would also like to thank Dr. Nicola Calabretta (Eindhoven University of Technology) and Dr. Domaniç Lavery (Infinera) for insightful discussions about passive optical networks.

\section{REFERENCES}

[1] G. Agrawal, Nonlinear Fiber Optics, 5th ed., Ser. Optics and Photonics. Boston: Academic Press, 2013.

[2] O. V. Sinkin, R. Holzlohner, J. Zweck, and C. R. Menyuk, "Optimization of the split-step Fourier method in modeling optical-fiber communications systems," J. Lightw. Technol., vol. 21, no. 1, pp. 61-68, Jan. 2003.

[3] L. Beygi, E. Agrell, M. Karlsson, and P. Johannisson, "Signal statistics in fiber-optical channels with polarization multiplexing and self-phase modulation," J. Lightw. Technol., vol. 29, no. 16, pp. 2379-2386, Aug. 2011.

[4] E. Forestieri and M. Secondini, "Solving the nonlinear schrödinger equation," in Proc. Opt. Commun. Theory Techn., 1st ed., E. Forestieri, Ed. Boston, USA: Springer, 2005, pp. 3-11.

[5] A. Vannucci, P. Serena, and A. Bononi, "The RP method: A new tool for the iterative solution of the nonlinear Schödinger equation," J. Lightw. Technol., vol. 20, no. 7, pp. 1102-1112, Jul. 2002.

[6] P. Poggiolini, G. Bosco, A. Carena, V. Curri, Y. Jiang, and F. Forghieri, "The GN-model of fiber non-linear propagation and its applications," $J$. Lightw. Technol., vol. 32, no. 4, pp. 694-721, 2014.

[7] A. Carena, G. Bosco, V. Curri, Y. Jiang, P. Poggiolini, and F. Forghieri, "EGN model of non-linear fiber propagation," Opt. Exp., vol. 22, no. 13, pp. 16335-16362, 2014.

[8] D. Semrau, R. I. Killey, and P. Bayvel, "The Gaussian noise model in the presence of inter-channel stimulated Raman scattering," J. Lightw. Technol., vol. 36, no. 14, pp. 3046-3055, Jul. 2018.

[9] W. Yan et al., "Low complexity digital perturbation back-propagation," in Proc. Eur. Conf. Exhib. Opt. Commun., 2011, pp. 1-3.

[10] E. Ciaramella and E. Forestieri, "Analytical approximation of nonlinear distortions," IEEE Photon. Technol. Lett., vol. 17, no. 1, pp. 91-93, Jan. 2005.

[11] V. Oliari, E. Agrell, and A. Alvarado, "Regular perturbation on the group-velocity dispersion parameter for nonlinear fibre-optical communications," Nature Commun., vol. 11, no. 933, pp. 1-11, Feb. 2020.

[12] V. Oliari, E. Agrell, and A. Alvarado, "Logarithmic perturbation models in the weak-dispersion regime with applications to passive optical networks," in Proc. Eur. Conf. Opt. Commun., 2020, pp. 1-4.

[13] M. Secondini and E. Forestieri, "Analytical fiber-optic channel model in the presence of cross-phase modulation," IEEE Photon. Technol. Lett., vol. 24, no. 22, pp. 2016-2019, Nov. 2012.

[14] M. Secondini, E. Forestieri, and G. Prati, "Achievable information rate in nonlinear WDM fiber-optic systems with arbitrary modulation formats and dispersion maps," J. Lightw. Technol., vol. 31, no. 23, pp. 3839-3852, Nov. 2013.

[15] M. Secondini, E. Agrell, E. Forestieri, D. Marsella, and M. R. Camara, "Nonlinearity mitigation in WDM systems: Models, strategies, and achievable rates," J. Lightw. Technol., vol. 37, no. 10, pp. 2270-2283, May 2019. 
[16] T. Koonen, "Fiber to the home/fiber to the premises: What, where, and when?" Proc. IEEE, vol. 94, no. 5, pp. 911-934, May 2006.

[17] B. De Vos et al., "Demonstration of extended split APON," in Proc. Opt. Fiber Commun. Conf. Exhibit, 2002, pp. 437-439.

[18] D. Piehler, "Implementing high [> 2048] split ratios in any PON," in Proc. Opt. Fiber Commun. Conf. Expo. Nat. Fiber Optic Engineers Conf., 2011, pp. 1-3.

[19] D. Lavery, M. Paskov, R. Maher, B. C. Thomsen, S. J. Savory, and P. Bayvel, "Low complexity multichannel nonlinear predistortion for passive optical networks," in Proc. Adv. Photon. Opt. Soc. Amer., 2015, Paper SpS2C.5

[20] 10-Gigabit-Capable Passive Optical Networks (XG-PON): Physical Media Dependent (PMD) Layer Specification, ITU-T G.987.2, Int. Telecommun. Union, Geneva, Switzerland, Feb. 2016.

[21] PON transmission technologies above $10 \mathrm{~Gb} / \mathrm{s}$ per wavelength, ITU-T G.Sup64, Int. Telecommun. Union, Geneva, Switzerland, Feb. 2018.

[22] D. Lavery, R. Maher, D. Millar, A. Alvarado, S. J. Savory, and P. Bayvel, "Why compensating fibre nonlinearity will never meet capacity demands," Dec. 2019, arXiv:1512.03426.

[23] 10-Gigabit-Capable Passive Optical Networks (XG-PON): Transmission Convergence (TC) Layer Specification, ITU-T G.987.3, Int. Telecommun. Union, Geneva, Switzerland, Jan. 2014.

[24] I. Reed and G. Solomon, "Polynomial codes over certain finite fields," $J$. Soc. Ind. Appl. Math., vol. 8, no. 2, pp. 300-304, Jun. 1960.

[25] L. Schmalen, A. J. de Lind van Wijngaarden, and S. Ten Brink, "Forward error correction in optical core and optical access networks," Bell Labs Tech. J., vol. 18, no. 3, pp. 39-66, 2013.

[26] B. Powell and K. Droskiewicz, "Latency \& Complexity for Various 25/50/100 G EPON FEC Code Proposals," Accessed: Aug. 20, 2020. [Online]. Available: https://www.ieee802.org/3/ca/public/meeting_ archive/2017/09/powell_3ca_1a_0917.pdf
[27] D. van Veen, V. Houtsma, A. de Lind van Wijngaarden, B. Powell, and E. Harstead, "FEC Code for 25/50/100 G EPON," Accessed Aug. 20, 2020. [Online]. Available: https://www.ieee802.org/3/ca/email/ pdfUGj1G0uq7U.pdf

[28] A. Teixeira et al., "DSP enabled optical detection techniques for PON," $J$ Lightw. Technol., vol. 38, no. 3, pp. 684-695, Feb. 2020.

[29] A. Alvarado, T. Fehenberger, B. Chen, and F. M. J. Willems, "Achievable information rates for fiber optics: Applications and computations," $J$. Lightw. Technol., vol. 36, no. 2, pp. 424-439, Jan. 2018.

[30] B. P. Smith, A. Farhood, A. Hunt, F. R. Kschischang, and J. Lodge, "Staircase codes: FEC for $100 \mathrm{~Gb} / \mathrm{s}$ OTN," J. Lightw. Technol., vol. 30 no. 1, pp. 110-117, Jan. 2012.

[31] Forward Error Correction for High Bit-Rate DWDM Submarine Systems, ITU-T G.975.1, Int. Telecommun. Union, Geneva, Switzerland, Feb. 2004

[32] T. Fehenberger, A. Alvarado, P. Bayvel, and N. Hanik, "On achievable rates for long-haul fiber-optic communications," Opt. Exp., vol. 23, no. 7 pp. 9183-9191, Apr. 2015.

[33] K. Atkinson, An Introduction to Numerical Analysis, 2nd ed. New York: Wiley, 1989

[34] M. Kamermans, "Gaussian quadrature weights and abscissae," Accessed: Jun. 23, 2021. [Online]. Available: https://pomax.github.io/bezierinfo/ legendre-gauss.html

[35] G. Bosco, A. Carena, V. Curri, R. Gaudino, P. Poggiolini, and S. Benedetto, "Suppression of spurious tones induced by the split-step method in fiber systems simulation," IEEE Photon. Technol. Lett., vol. 12, no. 5 , pp. 489-491, May 2000.

[36] V. Oliari, Frequency Logarithmic Perturbation on $\beta_{2}$. 2021, Accessed: Jun. 28, 2021. [Online]. Available: https://github.com/TUe-ICTLab/ Frequency-logarithmic-perturbation-on-beta2 